\title{
ANALYSIS OF SPECTRAL PROJECTORS IN ONE-DIMENSIONAL DOMAINS
}

\author{
Y. MADAY
}

\begin{abstract}
In this paper we analyze a class of projection operators with values in a subspace of polynomials. These projection operators are related to the Hilbert spaces involved in the numerical analysis of spectral methods. They are, in the first part of the paper, the standard Sobolev spaces and, in the second part, some weighted Sobolev spaces, the weight of which is related to the orthogonality relation satisfied by the Chebyshev polynomials. These results are used to study the approximation of a model fourth-order problem.
\end{abstract}

\section{INTRODUCTION}

The numerical analysis of the error between the exact and the numerical solutions of a partial differential equation approximated by spectral methods relies, in most cases, on the comparison between the numerical solution and the best polynomial approximation in some suitable Sobolev spaces. The best approximation is achieved by orthogonal projection operators. This paper presents an analysis of a wide class of such projection operators in weighted Sobolev spaces. The reason why this analysis is somewhat more difficult in spectral methods than in finite element methods has to do with the fact that the inverse inequality between spaces of polynomials provided with Sobolev norms is not as good as the ones that are available between spaces of finite elements. Because of this, the projection operators in low-order norm have poor approximation properties in higher norms (see Remark 2.1 of this paper).

Such projection operators have been studied before in $[4,12,16,1]$, but the existing results are limited to the approximation in low-order norms such as the $L^{2}$ - or $H^{1}$-norm and are therefore not adequate in many applications. For example, they are not sufficient for the error analysis of the approximation by spectral methods of fourth order and, in several instances, second-order problems (see [5]).

For different properties of projection operators that arise in spectral methods, see also [19].

An outline of the paper is as follows. In $\S 2$, we prove some results concerning the approximation theory with Legendre polynomials on the interval $(-1,1)$. These results are obtained in a standard Sobolev framework. The

Received June 14, 1985; revised June 16, 1987, October 13, 1989.

1980 Mathematics Subject Classification (1985 Revision). Primary 41A65; Secondary 65N30. 
theory of interpolation between Sobolev spaces is widely used here. In $\S 3$ we recall and complete results proved in $[6,1]$ concerning interpolation between weighted Sobolev spaces on $(-1,1)$. The weight is the one that appears in the orthogonality relation for Chebyshev polynomials. These properties are used in $\S 4$ to extend the results of $\S 2$ to the approximation theory with Chebyshev polynomials. Finally, we give in $\S 5$ an application to a simple fourth-order test problem. Note that other applications of these results can be found in $[3,18]$.

Let $\Delta$ be any interval of $\mathbb{R}$; for any integer $m$, the space of continuous functions defined on $\bar{\Delta}$ whose derivatives of order $\leq m$ are continuous on $\bar{\Delta}$ is denoted by $\mathscr{C}^{m}(\bar{\Delta})$; for any real number $s>0$, we denote by $\mathbf{H}^{s}(\Delta)$ the Sobolev space of index $s$ on $\Delta$ and by $\mathbf{H}_{0}^{s}(\Delta)$ the closure in $\mathbf{H}^{s}(\Delta)$ of the space $\mathscr{D}(\Delta)$ of all functions $\mathscr{C}^{\infty}$ with compact support in $\Delta$ (see [8] for more details). For any $s \geq 0$, we denote by $((\cdot, \cdot))_{s, \Delta}$ the scalar product of $\mathbf{H}^{s}(\Delta)$ and by $\|\cdot\|_{s, \Delta}$ its norm; the space $\mathbf{H}_{0}^{s}(\Delta)$ is provided with the standard seminorm $|\cdot|_{s, \Delta}$ of $\mathbf{H}^{s}(\Delta)$. In the sequel, the interval of reference will be the interval $\Lambda=(-1,1)$; for this interval, we shall omit the subscript $\Lambda$ in the various norms. For any function $f$, we denote by $f^{\prime}$ and $f^{\prime \prime}$ the first and second derivatives of $f$.

The duality pairing between a Hilbert space and its dual space is denoted by $\langle\cdot, \cdot\rangle$.

Let $\mathbf{X}$ and $\mathbf{Y}$ be two Hilbert spaces such that $\mathbf{X} \subset \mathbf{Y}$ and $\mathbf{X}$ is dense in $\mathbf{Y}$; for any $\theta$ in $[0,1]$, we denote by $[\mathbf{X}, \mathbf{Y}]_{\theta}$ the space obtained by any Hilbert interpolation of index $\theta$. We refer to [8, Chapter 1] for a complete analysis of Hilbert interpolation.

For any integer $N, \mathbb{P}_{N}$ stands for the space of all polynomials of degree $\leq N$ on $\Lambda$, and for any real number $s, s \geq 0$, we denote by $\mathbb{P}_{N}^{s}$ the space $\mathbb{P}_{N} \cap \mathbf{H}_{0}^{s}(\Lambda)$.

Finally, for any real number $s$, we denote by $\bar{s}$ the integral part of $s$.

\section{APPROXIMATION RESULTS FOR PROJECTION OPERATORS} IN THE STANDARD SOBOLEV SPACES

The main result of this section is

Theorem 2.1. Let $p$ and $s$ be two real numbers such that $p \notin \mathbb{N}+1 / 2,0 \leq$ $s \leq p$. There exists an operator $\Pi_{p, N}^{s, 0}$ from $\mathbf{H}^{p}(\Lambda) \cap \mathbf{H}_{0}^{s}(\Lambda)$ onto $\mathbb{P}_{N}^{s}$ such that, for any $\varphi \in \mathbf{H}^{\sigma}(\Lambda) \cap \mathbf{H}_{0}^{s}(\Lambda)$ with $\sigma \geq p$, we have

$$
\forall \nu, \quad 0 \leq \nu \leq p, \quad\left\|\varphi-\Pi_{p, N}^{s, 0} \varphi\right\|_{\nu} \leq C N^{\nu-\sigma}\|\varphi\|_{\sigma} .
$$

The proof of this theorem will be carried out in two steps. The first considers the case where $p$ is an integer, and the second generalizes the results to all values of $p$.

First step. Let us first consider the case where $p$ is equal to $s$ and belongs to $\mathbb{N}$. We define a projection operator $\mathbf{P}_{p, N}$ from $\mathbf{H}_{0}^{p}(\Lambda)$ onto $\mathbb{P}_{N}^{p}$ as follows: 
for any $\varphi \in \mathbf{H}_{0}^{p}(\Lambda), \mathbf{P}_{p, N}$ satisfies

$$
\forall \psi \in \mathbb{P}_{N}^{p}, \quad\left(\left(d^{p}\left[\varphi-\mathbf{P}_{p, N} \varphi\right] / d x^{p}, d^{p} \psi / d x^{p}\right)\right)_{0}=0 .
$$

This projection operator has the following properties.

Lemma 2.1. For any $\varphi \in \mathbf{H}^{\sigma}(\Lambda) \cap \mathbf{H}_{0}^{p}(\Lambda)$, with $\sigma \geq p$, we have

$$
\forall \nu, 0 \leq \nu \leq p, \quad\left\|\varphi-\mathbf{P}_{p, N} \varphi\right\|_{\nu} \leq C N^{\nu-\sigma}\|\varphi\|_{\sigma}
$$

Remark 2.1. The estimate in the case $\nu=p=0$ has been proved in [4], while the case $0 \leq \nu \leq p=1$ is analyzed in [12]. Moreover, it is proved in these two cases that no optimal bound is possible for $\mathbf{H}^{\nu}(\Lambda)$-norms with $\nu>p$. Indeed, the best estimate that can be achieved is

$$
\left\|\varphi-\mathbf{P}_{0, N} \varphi\right\|_{\nu} \leq C N^{2 \nu-\sigma}\|\varphi\|_{\sigma} .
$$

It is often necessary (see, e.g., $[2,3,18]$ ) to obtain optimal results in higher norms.

Proof of Lemma 2.1. We prove (2.2) for $\nu=p$ by induction over $p$ in $\mathbb{N}$. In Remark 2.1 we have recalled that the result is well known for $p=0$. Let us assume that, for any $\varphi$ in $\mathbf{H}^{\sigma}(\Lambda) \cap \mathbf{H}_{0}^{p-1}(\Lambda)$ with $\sigma \leq p-1$, we have

$$
\left\|\varphi-\mathbf{P}_{p-1, N} \varphi\right\|_{p-1} \leq C N^{p-1-\sigma}\|\varphi\|_{\sigma} .
$$

For techrical reasons that will be clarified in the sequel, we assume that

$$
N>2(p-1)
$$

(note that the asymptotic result $(2.2)$ is trivial for $N \leq 2(p-1)$ if we take the constant $C$ large enough). Let $\varphi$ be any element of $\mathbf{H}_{0}^{p}(\Lambda)$; then $\varphi^{\prime}$ belongs to $\mathbf{H}_{0}^{p-1}(\Lambda)$ and $\mathbf{P}_{p-1, N-1}\left(\varphi^{\prime}\right)$ is a polynomial in $\mathbb{P}_{N-1}^{p-1}$. Moreover, since $\varphi(-1)=\varphi(1)=0$, we have

$$
\int_{-1}^{1} \mathbf{P}_{p-1, N-1}\left(\varphi^{\prime}\right) d x=\int_{-1}^{1}\left[\mathbf{P}_{p-1, N-1}\left(\varphi^{\prime}\right)-\varphi^{\prime}\right] d x .
$$

Integrating by parts $2(p-1)$ times and noticing that $\mathbf{P}_{p-1, N-1}\left(\varphi^{\prime}\right)-\varphi^{\prime}$ belongs to $\mathbf{H}_{0}^{p-1}(\Lambda)$, we obtain

$$
\begin{aligned}
& \int_{-1}^{1} \mathbf{P}_{p-1, N-1}\left(\varphi^{\prime}\right) d x \\
& \quad=C(p)\left(\left(d^{(p-1)}\left[\mathbf{P}_{p-1, N-1}\left(\varphi^{\prime}\right)-\varphi^{\prime}\right] / d x^{(p-1)}, d^{(p-1)}\left[\left(1-x^{2}\right)^{p-1}\right] / d x^{(p-1)}\right)\right)_{0} .
\end{aligned}
$$

From (2.1), (2.4), we derive

$$
\int_{-1}^{1} \mathbf{P}_{p-1, N-1}\left(\varphi^{\prime}\right) d x=0 .
$$


Finally, we deduce that the primitive function $x \rightarrow \int_{-1}^{x} \mathbf{P}_{p-1, N-1}\left(\varphi^{\prime}\right)(y) d y=$ $R_{N}(x)$ is a polynomial of $\mathbb{P}_{N}^{p}$ that, for any $\psi$ in $\mathbb{P}_{N}^{p}$, satisfies

$$
\begin{aligned}
\left(\left(d^{p} R_{N} / d x^{p}, d^{p} \psi / d x^{p}\right)\right)_{0} & =\left(\left(d^{p-1}\left[\mathbf{P}_{p-1, N-1}\left(\varphi^{\prime}\right)\right] / d x^{p-1}, d^{p-1}\left[\psi^{\prime}\right] / d x^{p-1}\right)\right)_{0} \\
& =\left(\left(d^{p-1}\left[\varphi^{\prime}\right] / d x^{p-1}, d^{p-1}\left[\psi^{\prime}\right] / d x^{p-1}\right)\right)_{0} \\
& =\left(\left(d^{p} \varphi / d x^{p}, d^{p} \psi / d x^{p}\right)\right)_{0},
\end{aligned}
$$

so that $R_{N}=\mathbf{P}_{p, N} \varphi$. Then, using the classical Poincaré-Friedrichs inequality yields

$$
\left\|\varphi-\mathbf{P}_{p, N} \varphi\right\|_{p} \leq C\left\|\varphi^{\prime}-\left[\mathbf{P}_{p, N} \varphi\right]^{\prime}\right\|_{p-1}=C\left\|\varphi^{\prime}-\mathbf{P}_{p-1, N-1}\left(\varphi^{\prime}\right)\right\|_{p-1} .
$$

From the induction hypothesis we obtain

$$
\left\|\varphi-\mathbf{P}_{p, N} \varphi\right\|_{p} \leq C N^{p-1-(\sigma-1)}\left\|\varphi^{\prime}\right\|_{\sigma-1},
$$

so that

$$
\left\|\varphi-\mathbf{P}_{p, N} \varphi\right\|_{p} \leq C N^{p-\sigma}\|\varphi\|_{\sigma} .
$$

This proves the lemma for $\nu=p$.

In order to prove (2.2) for $\nu<p$, we use a duality argument. Consider the operator $\mathbf{L}_{p}$ defined from $\mathbf{H}^{-p}(\Lambda)$ into $\mathbf{H}_{0}^{p}(\Lambda)$ by

$$
\forall f \in \mathbf{H}^{-p}(\Lambda), \quad \forall \psi \in \mathbf{H}_{0}^{p}(\Lambda), \quad\left(\left(d^{p}\left(\mathbf{L}_{p} f\right) / d x^{p}, d^{p} \psi / d x^{p}\right)\right)_{0}=\langle f, \psi\rangle .
$$

It is well known that $\mathbf{L}_{p}$ is an isomorphism from $\mathbf{L}^{2}(\Lambda)$ into $\mathbf{H}^{2 p}(\Lambda) \cap \mathbf{H}_{0}^{p}(\Lambda)$, so that we have

$$
\begin{aligned}
\left\|\varphi-\mathbf{P}_{p, N} \varphi\right\|_{0} & =\sup _{\psi \in \mathbf{L}^{2}(\Lambda)} \frac{\left(\left(\varphi-\mathbf{P}_{p, N} \varphi, \psi\right)\right)_{0}}{\|\psi\|_{0}} \\
& =\sup _{\psi \in \mathbf{L}^{2}(\Lambda)} \frac{\left(\left(d^{p}\left(\varphi-\mathbf{P}_{p, N} \varphi\right) / d x^{p}, d^{p}\left(\mathbf{L}_{p} \psi\right) / d x^{p}\right)\right)_{0}}{\|\psi\|_{0}} \\
& =\sup _{\psi \in \mathbf{L}^{2}(\Lambda)} \frac{\left(\left(d^{p}\left(\varphi-\mathbf{P}_{p, N} \varphi\right) / d x^{p}, d^{p}\left(\mathbf{L}_{p} \psi-\mathbf{P}_{p, N}\left(\mathbf{L}_{p} \psi\right)\right) / d x^{p}\right)\right)_{0}}{\|\psi\|_{0}},
\end{aligned}
$$

where the last equality holds because of the definition (2.1) of $\mathbf{P}_{p, N} \varphi$. We deduce that

$$
\left\|\varphi-\mathbf{P}_{p, N} \varphi\right\|_{0} \leq\left\|\varphi-\mathbf{P}_{p, N} \varphi\right\|_{p} \sup _{\psi \in \mathbf{L}^{2}(\Lambda)} \frac{\left\|\mathbf{L}_{p} \psi-\mathbf{P}_{p, N}\left(\mathbf{L}_{p} \psi\right)\right\|_{p}}{\|\psi\|_{0}} ;
$$

using (2.6), we obtain

$$
\left\|\varphi-\mathbf{P}_{p, N} \varphi\right\|_{0} \leq C N^{p-\sigma}\|\varphi\|_{\sigma} N^{p-2 p} \sup _{\psi \in \mathbf{L}^{2}(\Lambda)} \frac{\left\|\mathbf{L}_{p} \psi\right\|_{2 p}}{\|\psi\|_{0}} .
$$

From the regularity of $\mathbf{L}_{p}$ we finally derive

$$
\left\|\varphi-\mathbf{P}_{p, N} \varphi\right\|_{0} \leq C N^{-\sigma}\|\varphi\|_{\sigma} .
$$


We have proved (2.2) for $\nu=0$ and $\nu=p$. We deduce now the result by interpolation. Indeed, the two results we have proved can also be stated as follows: the mapping Id $-\mathbf{P}_{p, N}$ is linear continuous

-from $\mathbf{H}^{\sigma}(\Lambda) \cap \mathbf{H}_{0}^{p}(\Lambda)$ into $\mathbf{L}^{2}(\Lambda)$ with a norm bounded by $C N^{-\sigma}$, -from $\mathbf{H}^{\sigma}(\Lambda) \cap \mathbf{H}_{0}^{p}(\Lambda)$ into $\mathbf{H}^{p}(\Lambda)$ with a norm bounded by $C N^{p-\sigma}$.

We derive from $[8$, Théorème 1.5.1] that, for any $\theta$ in $(0,1)$, the mapping Id $-\mathbf{P}_{p, N}$ is linear continuous

-from $\mathbf{H}^{\sigma}(\Lambda) \cap \mathbf{H}_{0}^{p}(\Lambda)$ into $\left[\mathbf{H}^{p}(\Lambda), \mathbf{L}^{2}(\Lambda)\right]_{\theta}=\mathbf{H}^{\theta p}(\Lambda)$ with a norm bounded by $C N^{\theta p-\sigma}$, which concludes the proof of (2.2).

We now turn to the case where $s$ and $p$ are integers such that $0 \leq s<p$. From the well-known properties of the traces we deduce that there exists a linear continuous operator $\mathbf{A}_{p}$ from $\mathbf{H}^{p}(\Lambda)$ into the subspace of polynomials $\mathbb{P}_{2 p-1}$ such that for any $\varphi$ in $\mathbf{H}^{p}(\Lambda) \cap \mathbf{H}_{0}^{s}(\Lambda)$ we have

$$
\begin{aligned}
& \varphi-\mathbf{A}_{p} \varphi \in \mathbf{H}_{0}^{p}(\Lambda) \quad \text { and } \quad\left\|\varphi-\mathbf{A}_{p} \varphi\right\|_{p} \leq C\|\varphi\|_{p}, \\
& \forall \mu \in \mathbb{R}^{+}, \quad\left\|\mathbf{A}_{p} \varphi\right\|_{\mu} \leq C(\mu)\|\varphi\|_{p} .
\end{aligned}
$$

It is an easy matter to check that, if we assume (2.4), the polynomial $\mathbf{A}_{p} \varphi+$ $\mathbf{P}_{p, N}\left(\varphi-\mathbf{A}_{p} \varphi\right)$ is an element of $\mathbb{P}_{N}^{s}$. Thanks to (2.2), it satisfies, for any $\sigma \geq p$ and any $\varphi$ in $\mathbf{H}^{\sigma}(\Lambda) \cap \mathbf{H}_{0}^{s}(\Lambda)$,

$$
\forall \nu, \quad 0 \leq \nu \leq p, \quad\left\|\varphi-\left(\mathbf{A}_{p} \varphi+\mathbf{P}_{p, N}\left(\varphi-\mathbf{A}_{p} \varphi\right)\right)\right\|_{\nu} \leq C N^{\nu-\sigma}\left\|\varphi-\mathbf{A}_{p} \varphi\right\|_{\sigma} .
$$

From (2.7) we deduce that

$$
\left\|\varphi-\left(\mathbf{A}_{p} \varphi+\mathbf{P}_{p, N}\left(\varphi-\mathbf{A}_{p} \varphi\right)\right)\right\|_{\nu} \leq C N^{\nu-\sigma}\|\varphi\|_{\sigma} .
$$

We now define $\Pi_{p, N}^{s, 0}$ to be the projection operator from $\mathbf{H}^{p}(\Lambda) \cap \mathbf{H}_{0}^{s}(\Lambda)$ onto $\mathbb{P}_{N}^{s}$ with respect to the scalar product $((\cdot, \cdot))_{p}$ of $\mathbf{H}^{p}(\Lambda)$. Using this last inequality with $\nu=p$, it is an easy matter to check that

$$
\left\|\varphi-\Pi_{p, N}^{s, 0} \varphi\right\|_{p}=\inf _{\varphi_{N} \in \mathbb{P}_{N}^{s}}\left\|\varphi-\varphi_{N}\right\|_{p} \leq\left\|\varphi-\left(\mathbf{A}_{p} \varphi+\mathbf{P}_{p, N}\left(\varphi-\mathbf{A}_{p} \varphi\right)\right)\right\|_{p},
$$

so that

$$
\left\|\varphi-\Pi_{p, N}^{s, 0} \varphi\right\|_{p} \leq C N^{p-\sigma}\|\varphi\|_{\sigma} .
$$

We now use a duality argument to analyze $\left\|\varphi-\Pi_{p, N}^{s, 0} \varphi\right\|_{0}$. As in the case $s=p$, consider the operator $\tilde{\mathbf{L}}_{p}$ defined from $\mathbf{H}^{-p}(\Lambda)$ into $\mathbf{H}_{0}^{p}(\Lambda)$ by

$$
\forall f \in \mathbf{H}^{-p}(\Lambda), \forall \psi \in \mathbf{H}_{0}^{p}(\Lambda), \quad\left(\left(\tilde{\mathbf{L}}_{p} f, \psi\right)\right)_{p}=\langle f, \psi\rangle .
$$

It is well known that $\tilde{\mathbf{L}}_{p}$ is an isomorphism from $\mathbf{L}^{2}(\Lambda)$ into $\mathbf{H}^{2 p}(\Lambda) \cap \mathbf{H}_{0}^{p}(\Lambda)$, 
so that we have

$$
\begin{aligned}
\left\|\varphi-\Pi_{p, N}^{s, 0} \varphi\right\|_{0} & =\sup _{\psi \in \mathbf{L}^{2}(\Lambda)} \frac{\left(\left(\varphi-\Pi_{p, N}^{s, 0} \varphi, \psi\right)\right)_{0}}{\|\psi\|_{0}} \\
& =\sup _{\psi \in \mathbf{L}^{2}(\Lambda)} \frac{\left(\left(\left(\varphi-\Pi_{p, N}^{s, 0} \varphi\right), \tilde{\mathbf{L}}_{p} \psi\right)\right)_{p}}{\|\psi\|_{0}} \\
& =\sup _{\psi \in \mathbf{L}^{2}(\Lambda)} \frac{\left(\left(\left(\varphi-\Pi_{p, N}^{s, 0} \varphi\right),\left(\tilde{\mathbf{L}}_{p} \psi-\Pi_{p, N}^{s, 0}\left(\tilde{\mathbf{L}}_{p} \psi\right)\right)\right)\right)_{p}}{\|\psi\|_{0}},
\end{aligned}
$$

where the last equality holds because of the definition of $\Pi_{p, N}^{s, 0} \varphi$. We deduce that

$$
\left\|\varphi-\Pi_{p, N}^{s, 0} \varphi\right\|_{0} \leq\left\|\varphi-\Pi_{p, N}^{s, 0} \varphi\right\|_{p} \sup _{\psi \in \mathbf{L}^{2}(\Lambda)} \frac{\left\|\tilde{\mathbf{L}}_{p} \psi-\Pi_{p, N}^{s, 0}\left(\tilde{\mathbf{L}}_{p} \psi\right)\right\|_{p}}{\|\psi\|_{0}}
$$

using (2.8), we obtain

$$
\left\|\varphi-\Pi_{p, N}^{s, 0} \varphi\right\|_{0} \leq C N^{p-\sigma}\|\varphi\|_{\sigma} N^{p-2 p} \sup _{\psi \in \mathbf{L}^{2}(\Lambda)} \frac{\left\|\tilde{\mathbf{L}}_{p} \psi\right\|_{2 p}}{\|\psi\|_{0}} .
$$

From the regularity of $\widetilde{\mathbf{L}}_{p}$ we finally derive

$$
\left\|\varphi-\Pi_{p, N}^{s, 0} \varphi\right\|_{0} \leq C N^{-\sigma}\|\varphi\|_{\sigma} .
$$

With an interpolation result we then obtain an estimate for $\left\|\varphi-\Pi_{p, N}^{s, 0} \varphi\right\|_{\nu}$ for any $\nu, 0 \leq \nu \leq p$. Hence, we have proved

Lemma 2.2. For any $\varphi \in \mathbf{H}^{\sigma}(\Lambda) \cap \mathbf{H}_{0}^{s}(\Lambda)$, with $\sigma \geq p$, we have

$$
\forall \nu, \quad 0 \leq \nu \leq p, \quad\left\|\varphi-\Pi_{p, N}^{s, 0} \varphi\right\|_{\nu} \leq C N^{\nu-\sigma}\|\varphi\|_{\sigma} .
$$

Second step. We want to extend Lemma 2.1 to nonintegral values of $p, p \notin$ $\mathbb{N}+1 / 2$. We must distinguish the two cases $p-\bar{p}<1 / 2$ and $p-\bar{p}>1 / 2$.

We begin with the case $p-\bar{p}>1 / 2$ and take $\varphi$ in $\mathbf{H}^{\sigma}(\Lambda) \cap \mathbf{H}_{0}^{p}(\Lambda)$ with $\sigma \geq \bar{p}+1$. It is standard to note that $\varphi$ is, in fact, in $\mathbf{H}_{0}^{\bar{p}+1}(\Lambda)$. Now using Lemma 2.1, we obtain

$$
\inf _{\varphi_{N} \in \mathbb{P}_{N}^{\bar{p}+1}}\left\|\varphi-\varphi_{N}\right\|_{p} \leq C N^{p-\sigma}\|\varphi\|_{\sigma}
$$

for any $\sigma \geq \bar{p}+1$ (use (2.2) for $\mathbf{P}_{\bar{p}+1, N}$ in the case $\nu=p$ ). Since we have $\mathbb{P}_{N}^{\bar{p}+1}=\mathbb{P}_{N}^{p}$, we deduce that

$$
\forall \sigma \geq \bar{p}+1, \quad \inf _{\varphi_{N} \in \mathbb{P}_{N}^{p}}\left\|\varphi-\varphi_{N}\right\|_{p} \leq C N^{p-\sigma}\|\varphi\|_{\sigma}
$$

We now consider the case $p-\bar{p}<1 / 2$ and take $\varphi \in \mathbf{H}^{\sigma}(\Lambda) \cap \mathbf{H}_{0}^{p}(\Lambda)$, $\sigma \geq \bar{p}+1$. Using now Lemma 2.2 gives

$$
\inf _{\varphi_{N} \in \mathbb{P}_{N}^{\bar{p}}}\left\|\varphi-\varphi_{N}\right\|_{p} \leq C N^{p-\sigma}\|\varphi\|_{\sigma}
$$


(use (2.10) for $\Pi_{\bar{p}+1, N}^{\bar{p}, 0}$ with $\nu=p$ ), so that, for any $\sigma \geq \bar{p}+1$,

$$
\forall \sigma \geq \bar{p}+1, \quad \inf _{\varphi_{N} \in \mathbb{P}_{N}^{p}}\left\|\varphi-\varphi_{N}\right\|_{p} \leq C N^{p-\sigma}\|\varphi\|_{\sigma} .
$$

Now define, for nonintegral values of $p, p \notin \mathbb{N}+1 / 2$, a scalar product over $\mathbf{H}_{0}^{p}(\Lambda)$ obtained by interpolation of index $1 / 2$ between $L^{2}(\Lambda)$ and $\mathbf{H}_{0}^{2 p}(\Lambda)$ if $2 p$ does not belong to $\mathbb{N}+1 / 2$, and by interpolation of index $1 / 3$ between $\mathbf{L}^{2}(\Lambda)$ and $\mathbf{H}_{0}^{3 p}(\Lambda)$ if $2 p$ belongs to $\mathbb{N}+1 / 2$ (see [8, p. 12 and Chapter 1 , $\S 11.5])$. More precisely, we consider the domain operator interpolation: there exists a self-adjoint operator $\Theta_{p}$ such that

-the domain $D\left(\boldsymbol{\Theta}_{p}\right)$ of the operator $\Theta_{p}$ in $\mathbf{L}^{2}(\Lambda)$ is $\mathbf{H}_{0}^{p}(\Lambda)$,

-if $2 p$ does not belong to $\mathbb{N}+1 / 2$, the domain $\mathbf{D}\left(\Theta_{p}^{2}\right)$ of the operator $\Theta_{p}^{2}$ in $\mathbf{L}^{2}(\Lambda)$ is $\mathbf{H}_{0}^{2 p}(\Lambda)$; if $2 p$ belongs to $\mathbb{N}+1 / 2$, the domian $\mathbf{D}\left(\Theta_{p}^{3}\right)$ of the operator $\Theta_{p}^{3}$ in $\mathbf{L}^{2}(\Lambda)$ is $\mathbf{H}_{0}^{3 p}(\Lambda)$. Moreover, if $p$ does not belong to $\mathbb{N}+1 / 2$, the bilinear form

$$
(u, v) \rightarrow(((u, v)))_{p}=((u, v))_{0}+\left(\left(\boldsymbol{\Theta}_{p} u, \boldsymbol{\Theta}_{p} v\right)\right)_{0}
$$

is a scalar product whose associated norm, denoted by ||$\cdot|\||_{p}$, is equivalent to the one defined in (2.2).

From (2.11) and (2.12), the projection operator $\mathbf{P}_{p, N}$ from $\mathbf{H}_{0}^{p}(\Lambda)$, provided with the norm $\|\mid \cdot\|_{p}$, onto $\mathbb{P}_{N}^{p}$ verifies the following estimate, for any $\varphi \in$ $\mathbf{H}^{\sigma}(\Lambda) \cap \mathbf{H}_{0}^{p}(\Lambda), \sigma \geq \bar{p}+1$,

$$
\left\|\varphi-\mathbf{P}_{p, N} \varphi\right\|_{p} \leq C N^{p-\sigma}\|\varphi\|_{\sigma} .
$$

Trivially, we also have

$$
\left\|\varphi-\mathbf{P}_{p, N} \varphi\right\|_{p} \leq C\|\varphi\|_{p} .
$$

A simple interpolation argument now gives, for any $\sigma \geq p$ and any $\varphi \in \mathbf{H}^{\sigma}(\Lambda) \cap$ $\mathbf{H}_{0}^{p}(\Lambda)$,

$$
\left\|\varphi-\mathbf{P}_{p, N} \varphi\right\|_{p} \leq C N^{p-\sigma}\|\varphi\|_{\sigma} .
$$

We now use the abstract duality result of the appendix to derive an optimal estimate for $\left\|\varphi-\mathbf{P}_{p, N} \varphi\right\|_{0}$. In the situation where $\mathbf{Y}_{0}=\mathbf{L}^{2}(\Lambda), \mathbf{Z}=\mathbf{H}^{2 p}(\Lambda) \cap$ $\mathbf{H}_{0}^{p}(\Lambda), \mathbf{Y}=\mathbf{D}\left(\boldsymbol{\Theta}_{p}^{2}\right)$, and $\mathbf{X}=\mathbf{H}_{0}^{p}(\Lambda)$, we obtain from (2.14) that

$$
\begin{aligned}
\left\|\varphi-\mathbf{P}_{p, N} \varphi\right\|_{0} & \leq C N^{p-\sigma}\|\varphi\|_{\sigma} \sup _{\psi \in \mathbf{H}^{2 p}(\Lambda) \cap \mathbf{H}_{0}^{p}(\Lambda)} \frac{\left\|\psi-\mathbf{P}_{p, N} \psi\right\|_{p}}{\|\psi\|_{2 p}} \\
& \leq C N^{-\sigma}\|\varphi\|_{\sigma} .
\end{aligned}
$$

Interpolating between (2.14) and the previous estimate, we conclude that, for any $\varphi \in \mathbf{H}^{\sigma}(\Lambda) \cap \mathbf{H}_{0}^{p}(\Lambda), \sigma \geq p$, we have

$$
\forall \nu, \quad 0 \leq \nu \leq p, \quad\left\|\varphi-\mathbf{P}_{p, N} \varphi\right\|_{\nu} \leq C N^{\nu-\sigma}\|\varphi\|_{\sigma} .
$$


Following the same lines as in the proof of Lemma 2.2, we deduce from the result (2.15) the existence of an operator $\Pi_{p, N}^{s, 0}$ for any values of $p$ and $s$ that satisfy the bound of Theorem 2.1.

Remark 2.2. It is an easy matter to state a similar result concerning the existence of an operator from the closed subspace of all elements of $\mathbf{H}^{p}(\Lambda)$ such that only some of the derivatives of order $<p-1 / 2$ vanish on the boundary of $\Lambda$ onto the space of polynomials in $\mathbb{P}_{N}$ having the same derivatives equal to zero at the boundary.

Remark 2.3. As pointed out in [18], the operator $\mathbf{A}_{p}+\mathbf{P}_{p, N} \circ\left(\mathrm{Id}-\mathbf{A}_{p}\right)$ defined in (2.7) for integral values of $p$, and that could be defined in a similar way for any real number $p$ not in $\mathbb{N}+1 / 4$, has the same approximation properties as $\Pi_{p, N}$ and, moreover, it preserves the traces of any element of $\mathbf{H}^{p}(\Lambda)$.

\section{SOME RESUlTS CONCERNING Weighted SOBOLEV SPACES}

3.1. Notation and basic properties. Let us briefly recall the definition of the weighted Sobolev spaces we shall use. If $\rho(x)=\left(1-x^{2}\right)^{-1 / 2}$ denotes the Chebyshev weight on the interval $\Lambda$, let

$$
\mathbf{L}_{\rho}^{2}(\Lambda)=\left\{\Phi: \Lambda \rightarrow \mathbb{R} \mid \Phi \text { is measurable and } \int_{\Lambda} \Phi^{2}(x) \rho(x) d x<+\infty\right\}
$$

be the Lebesgue space associated with the weight $\rho(x)$. We provide it with the inner product

$$
(\Phi, \Psi)_{\rho}=\int_{\Lambda} \Phi(x) \Psi(x) \rho(x) d x .
$$

For any integer $m \geq 1$, we set

$$
\mathbf{H}_{\rho}^{m}(\Lambda)=\left\{\boldsymbol{\Phi} \in \mathbf{L}_{\rho}^{2}(\Lambda) \mid d^{k} \boldsymbol{\Phi} / d x^{k} \in \mathbf{L}_{\rho}^{2}(\Lambda), \quad 1 \leq k \leq m\right\},
$$

the weighted Sobolev space of order $m$, provided with the inner product

$$
((\boldsymbol{\Phi}, \Psi))_{m, \rho}=\sum_{k=0}^{m}\left(d^{k} \Phi / d x^{k}, d^{k} \Psi / d x^{k}\right)_{\rho}
$$

and the norm

$$
\|\Phi\|_{m, \rho}^{2}=((\Phi, \Phi))_{m, \rho} .
$$

For any real number $s=m+\alpha$, with $0<\alpha<1$ and $m$ an integer, $\mathbf{H}_{\rho}^{s}(\Lambda)$ is defined by Hilbert interpolation of index $\alpha$ between the space $\mathbf{H}_{\rho}^{m}(\Lambda)$ and $\mathbf{H}_{\rho}^{m+1}(\Lambda)$ :

$$
\mathbf{H}_{\rho}^{s}(\Lambda)=\left[\mathbf{H}_{\rho}^{m}(\Lambda), \mathbf{H}_{\rho}^{m+1}(\Lambda)\right]_{\alpha}
$$

(see [8] for more details). In addition, we define $\mathbf{H}_{\rho, 0}^{s}(\Lambda)$ to be the closure of $\mathscr{D}(\Lambda)$ in $\mathbf{H}_{\rho}^{s}(\Lambda)$.

We recall some results proved in [1] that extend to these spaces the results of [6]. 
Theorem 3.1. (i) For any real numbers $s, p, q$ such that $0 \leq q<s<p$ and which do not belong to $\mathbb{N}+1 / 4$,

$$
\mathbf{H}_{\rho, 0}^{s}(\Lambda)=\left[\mathbf{H}_{\rho, 0}^{p}(\Lambda), \mathbf{H}_{\rho, 0}^{q}(\Lambda)\right]_{\theta},
$$

with $\theta=(s-p) /(q-p)$.

(ii) For any real numbers $s, p, q$ such that $0 \leq q<s<p$ and such that $p$ and $q$ do not belong to $\mathbb{N}+1 / 4, \mathbf{H}_{\rho}^{s}(\Lambda)$ satisfies the following topological imbedding:

$$
\mathbf{H}_{\rho}^{s}(\Lambda) \subset\left[\mathbf{H}_{\rho}^{p}(\Lambda), \mathbf{H}_{\rho}^{q}(\Lambda)\right]_{\theta},
$$

with $\theta=(s-p) /(q-p)$.

(iii) For any real number $s>1 / 2, s \notin \mathbb{N}+1 / 2$, the space $\mathbf{H}_{\rho}^{s}(\Lambda)$ is contained in $\mathbf{C}^{\nu}(\bar{\Lambda})$, where $\nu$ is the integral part of $s-1 / 2$. by

(iv) For any integer $m$, the trace mapping defined from $\mathbf{C}^{\infty}(\bar{\Lambda})$ into $\mathbb{R}^{2 m+2}$

$$
\begin{aligned}
u \rightarrow\left(u(-1), d u / d x(-1), \ldots, d^{m} u / d x^{m}(-1),\right. \\
\left.u(1), d u / d x(1), \ldots, d^{m} u / d x^{m}(1)\right),
\end{aligned}
$$

can be extended to a continuous mapping from $\mathbf{H}_{\rho}^{s}(\Lambda)$ onto $\mathbb{R}^{2 m+2}$ for any real number $s>m+1 / 4$. Moreover, the space $\mathbf{H}_{\rho, 0}^{s}(\Lambda)$ coincides with the kernel of that trace mapping.

We recall also the following result that can be found in [5].

Theorem 3.2. For any real number $s \geq 1 / 4, \mathbf{H}^{s}(\Lambda)$ is contained in $\mathbf{H}_{\rho}^{s-1 / 4}(\Lambda)$.

3.2. The case of real interpolation. Let us first use a wider class of interpolation that depends on two real numbers. It consists of the real interpolation procedure (see [9] for more details), and we recall that for all $\theta \in(0,1)$, and $\left(r, r^{\prime}\right) \in$ $\left(\overline{\mathbb{R}}^{+}\right)^{2}, r^{\prime} \geq r$,

$$
\left[\mathbf{H}_{\rho}^{p}(\Lambda), \mathbf{H}_{\rho}^{q}(\Lambda)\right]_{\theta, r} \subset\left[\mathbf{H}_{\rho}^{p}(\Lambda), \mathbf{H}_{\rho}^{q}(\Lambda)\right]_{\theta, r^{\prime}}
$$

and that, in the case where $r$ is equal to 2 , this interpolation coincides with any Hilbert interpolation.

Define the weight function $\omega$ on $\mathbb{R}^{+}$by

$$
\forall x \in \mathbb{R}^{+}, \quad \omega(x)= \begin{cases}x^{-1 / 2}, & 0 \leq x \leq 1, \\ 1, & x \geq 1\end{cases}
$$

We define the weighted Sobolev spaces associated with this weight as follows:

$$
\mathbf{L}_{\omega}^{2}\left(\mathbb{R}^{+}\right)=\left\{\Phi: \mathbb{R}^{+} \rightarrow \mathbb{R} \mid \Phi \text { is measurable and } \int_{\mathbb{R}^{+}} \Phi^{2}(x) \omega(x) d x<+\infty\right\},
$$

and, for any integer $m \geq 1$,

$$
\mathbf{H}_{\omega}^{m}\left(\mathbb{R}^{+}\right)=\left\{\Phi \in \mathbf{L}_{\omega}^{2}\left(\mathbb{R}^{+}\right) \mid d^{k} \Phi / d x^{k} \in \mathbf{L}_{\omega}^{2}\left(\mathbb{R}^{+}\right), \quad 1 \leq k \leq m\right\}
$$

these spaces are provided with the norm $\|\cdot\|_{m, \omega}$.

The next lemma exhibits an equivalent norm over $\mathbf{H}_{\omega}^{n}\left(\mathbb{R}^{+}\right)$. 
Lemma 3.1. For any integer $n \geq 2$ and for any $u$ in $\mathbf{H}_{\omega}^{n}\left(\mathbb{R}^{+}\right)$we have

$$
\forall m, 0<m<n, \quad\left\|d^{m} u / d x^{m}\right\|_{0, \omega} \leq C\left(\|u\|_{0, \omega}+\left\|d^{n} u / d x^{n}\right\|_{0, \omega}\right) .
$$

Proof. Let us choose a function $u$ in $\mathbf{H}_{\omega}^{n}\left(\mathbb{R}^{+}\right)$, and define $u^{*}$ as the orthogonal projection of $u_{\mid(0,1)}$ into $\mathbb{P}_{n}$ for the $\mathbf{L}^{2}(0,1)$ scalar product. It satisfies

$$
\forall \varphi \in \mathbb{P}_{n}, \quad \int_{0}^{1}\left(u-u^{*}\right)(x) \varphi(x) d x=0,
$$

and, from the uniform equivalence of norms over $\mathbb{P}_{n}$,

$$
\forall \mu>0, \quad\left\|u^{*}\right\|_{\mu,(0,1)} \leq C(n)\|u\|_{0, \omega} .
$$

We deduce that, in particular, the element $\bar{u}=u-u^{*}$ has zero average and is continuous on $(0,1)$, hence $\bar{u}$ vanishes at least once in $(0,1)$. If $\bar{u}$ has a finite number of zeros in $(0,1)$, let $\left(\xi_{i}\right)_{1 \leq i \leq p}$ be all of those with odd multiplicity. It is an easy consequence of the definition of the $\xi_{i}$ to note that $(\bar{u})\left(\prod_{i=1}^{p}\left(x-\xi_{i}\right)\right)$ has constant sign on the interval $(0,1)$. Let us assume that $p<n$; taking $\varphi=\prod_{i=1}^{p}\left(x-\xi_{i}\right)$ in (3.9) implies that $(\bar{u})\left(\prod_{i=1}^{p}\left(x-\xi_{i}\right)\right)$ is constant equal to 0 on $(0,1)$, which contradicts the assumption. We therefore deduce that $\bar{u}$ has at least $n$ distinct zeros in $(0,1)$. This implies that for any $l, 1 \leq l \leq n-1$, the function $\partial^{l}(\bar{u}) / \partial x^{l}$ vanishes at least at one point $\mu_{l}$ in $(0,1)$. As a consequence, we note that

$$
\begin{aligned}
\forall y \in[0,1], \quad\left|\left(\partial^{l} \bar{u} / \partial x^{l}\right)(y)\right| & \leq\left|\int_{\mu_{l}}^{y} \partial^{l+1} \bar{u} / \partial x^{l+1}(t) d t\right| \\
& \leq C\left[\int_{0}^{1}\left(\partial^{l+1} \bar{u} / \partial x^{l+1}\right)^{2} \omega(y) d y\right]^{1 / 2},
\end{aligned}
$$

whence, from (3.10), we deduce that

$$
\begin{aligned}
\forall y \in[0,1], \quad & \left|\left(\partial^{l} \bar{u} / \partial x^{l}\right)(y)\right| \\
& \leq C\left(\|u\|_{0, \omega}+\left[\int_{0}^{1}\left(\partial^{l+1} \bar{u} / \partial x^{l+1}\right)^{2} \omega(y) d y\right]^{1 / 2}\right)
\end{aligned}
$$

and

$$
\int_{0}^{1}\left(\partial^{l} \bar{u} / \partial x^{l}\right)^{2} \omega(y) d y \leq C\left(\|u\|_{0, \omega}+\left[\int_{0}^{1}\left(\partial^{l+1} \bar{u} / \partial x^{l+1}\right)^{2} \omega(y) d y\right]^{1 / 2}\right) .
$$

By induction, we derive that

$$
\begin{aligned}
\forall l, 1 \leq l \leq n-1, \int_{0}^{1}\left(\partial^{l} u / \partial x^{l}\right)^{2}(y) \omega(y) d y \\
\leq C\left(\|u\|_{0, \omega}+\left\|\partial^{n} u / \partial x^{n}\right\|_{0, \omega}\right) .
\end{aligned}
$$

Let $\nu$ be an element of $\mathscr{C}^{\infty}\left(\mathbb{R}^{+}\right)$with values in $[0,1]$, with compact support in $[0,3]$, and such that $\nu(1)=1$. The element $\widetilde{u}$ defined on $\mathbb{R}^{+}$by

$$
\forall x>0, \quad \tilde{u}(x)=u(x)-\left[\sum_{l=1}^{n-1}(x-1)^{l} \nu(x) u^{(l)}(1) / l !\right]
$$


satisfies

$$
\forall l, \quad 1 \leq l \leq n-1, \quad \partial^{l} \tilde{u} / \partial x^{l}(1)=0 ;
$$

moreover, we derive from (3.11) that

(3.14) $\forall l, 1 \leq l \leq n-1, \quad\left\|\partial^{l}(\tilde{u}-u) / \partial x^{l}\right\|_{n, \omega} \leq C\left(\|u\|_{0, \omega}+\left\|\partial^{n} u / \partial x^{n}\right\|_{0, \omega}\right)$.

Integrating by parts and using (3.13), we deduce that

$$
\begin{aligned}
\forall l, \quad 1 \leq l \leq n-1, \quad \int_{1}^{\infty}\left(\partial^{l} \tilde{u} / \partial x^{l}\right)^{2}(x) d x \\
=-\int_{1}^{\infty}\left(\partial^{l-1} \tilde{u} / \partial x^{l-1}\right)\left(\partial^{l+1} \tilde{u} / \partial x^{l+1}\right)(x) d x
\end{aligned}
$$

hence $\forall l, \quad 1 \leq l \leq n-1$,

$$
\begin{aligned}
\int_{1}^{\infty}\left(\partial^{l} \tilde{u} / \partial x^{l}\right)^{2}(x) d x \leq & \frac{1}{2} \int_{1}^{\infty}\left(\partial^{l-1} \tilde{u} / \partial x^{l-1}\right)^{2}(x) d x \\
& +\frac{1}{2} \int_{1}^{\infty}\left(\partial^{l+1} \tilde{u} / \partial x^{l+1}\right)^{2}(x) d x .
\end{aligned}
$$

Iterating this process, we find that

$$
\begin{aligned}
\forall l, \quad 1 \leq l \leq n-1, \quad \int_{1}^{\infty}\left(\partial^{l} \tilde{u} / \partial x^{l}\right)^{2}(x) d x \\
\quad \leq C\left[\int_{1}^{\infty}(\tilde{u})^{2}(x) d x+\int_{1}^{\infty}\left(\partial^{n} \tilde{u} / \partial x^{n}\right)^{2}(x) d x\right],
\end{aligned}
$$

and from (3.14), we conclude

$$
\begin{aligned}
\forall m, 1 \leq m \leq n-1, \quad \int_{1}^{\infty} & \left(\partial^{m} u / \partial x^{m}\right)^{2}(y) \omega(y) d y \\
& \leq C\left(\|u\|_{0, \omega}+\left\|\partial^{n} u / \partial x^{n}\right\|_{0, \omega}\right) .
\end{aligned}
$$

This last result and (3.12) yield the lemma.

Corollary 3.1. For any function $u$ in $\mathbf{H}_{\omega}^{2}\left(\mathbb{R}^{+}\right)$we have

$$
\|u\|_{1, \omega}^{2} \leq C\|u\|_{0, \omega}\|u\|_{2, \omega} \text {. }
$$

Proof. Applying (3.8), with $n=2$, to the function $x \rightarrow u(t x)$, for $t>0$, we derive that

$$
\forall t>0, \quad t\left\|u^{\prime}\right\|_{0, \omega}-C\left(\|u\|_{0, \omega}+t^{2}\left\|u^{\prime \prime}\right\|_{0, \omega}\right) \leq 0
$$

If the roots of the polynomial $\left[t\left\|u^{\prime}\right\|_{0, \omega}-C\left(\|u\|_{0, \omega}+t^{2}\left\|u^{\prime \prime}\right\|_{0, \omega}\right)\right]$ are real, they are positive; hence, we deduce from (3.16) that the discriminant of this polynomial is nonpositive, that is,

$$
\left\|u^{\prime}\right\|_{0, \omega}^{2}-4 C^{2}\|u\|_{0, \omega}\left\|u^{\prime \prime}\right\|_{0, \omega} \leq 0
$$

This immediately yields (3.15). 
Another consequence of Lemma 3.1 is

Corollary 3.2. Let $n$ be an integer; the scalar product on $\mathbf{H}_{\rho}^{n}(\Lambda)$,

$$
((\varphi, \psi))_{n, \rho}=(\varphi, \psi)_{0, \rho}+\left(d^{n}(\varphi) / d x^{n}, d^{n}(\psi) / d x^{n}\right)_{0, \rho}
$$

defines a norm on $\mathbf{H}_{\rho}^{n}(\Lambda)$ equivalent to the standard one.

Let $\mathbf{X}$ be a Banach space. We say that $\mathbf{X}$ is contained in $\mathscr{K}_{\theta}\left(\mathbf{H}_{\rho}^{p}(\Lambda), \mathbf{H}_{\rho}^{q}(\Lambda)\right)$ for a real number $\theta$ in $(0,1)$ if

$$
\left[\mathbf{H}_{\rho}^{p}(\Lambda), \mathbf{H}_{\rho}^{q}(\Lambda)\right]_{\theta, 1} \subset \mathbf{X} \subset\left[\mathbf{H}_{\rho}^{p}(\Lambda), \mathbf{H}_{\rho}^{q}(\Lambda)\right]_{\theta, \infty} .
$$

We recall the following characterization: a Banach space $\mathbf{X}$ contains the space $\left[\mathbf{H}_{\rho}^{p}(\Lambda), \mathbf{H}_{\rho}^{q}(\Lambda)\right]_{\theta, 1}$ if and only if

$$
\forall u \in \mathbf{H}_{\rho}^{p}(\Lambda), \quad\|u\|_{\mathbf{X}} \leq C\|u\|_{p, \rho}^{(1-\theta)}\|u\|_{q, \rho}^{\theta} .
$$

We now prove

Lemma 3.2. The space $\mathbf{H}_{\rho}^{1}(\Lambda)$ is contained in $\mathscr{K}_{1 / 2}\left(\mathbf{H}_{\rho}^{2}(\Lambda), \mathbf{L}_{\rho}^{2}(\Lambda)\right)$.

Proof. By (3.6) and (3.7), we immediately obtain

$$
\mathbf{H}_{\rho}^{1}(\Lambda) \subset\left[\mathbf{H}_{\rho}^{2}(\Lambda), \mathbf{L}_{\rho}^{2}(\Lambda)\right]_{1 / 2, \infty} .
$$

Let us consider a function $\beta$ of $\mathbf{C}^{\infty}(\bar{\Lambda})$ with values in $[0,1]$ such that

$$
\begin{aligned}
& \forall x, \quad-1 \leq x \leq-1 / 3, \quad \beta(x)=1, \\
& \forall x, \quad 1 / 3 \leq x \leq 1, \quad \beta(x)=0 .
\end{aligned}
$$

Let $w$ be any element of $\mathbf{H}_{\rho}^{2}(\Lambda)$. We derive from (3.15) that

$$
\begin{aligned}
& \|\beta w\|_{1, \rho}^{2} \leq C\|\beta w\|_{0, \rho}\|\beta w\|_{2, \rho} \leq C\|w\|_{0, \rho}\|w\|_{2, \rho}, \\
& \|(1-\beta) w\|_{1, \rho}^{2} \leq C\|(1-\beta) w\|_{0, \rho}\|(1-\beta) w\|_{2, \rho} \leq C\|w\|_{0, \rho}\|w\|_{2, \rho} .
\end{aligned}
$$

From the Cauchy-Schwarz inequality, we then obtain

$$
\forall w \in \mathbf{H}_{\rho}^{2}(\Lambda), \quad\|w\|_{1, \rho} \leq\|\beta w\|_{1, \rho}+\|(1-\beta) w\|_{1, \rho} \leq C\|w\|_{0, \rho}^{1 / 2}\|w\|_{2, \rho}^{1 / 2} .
$$

The lemma now follows from (3.19) and (3.20).

We can easily extend the same techniques to higher derivatives and state

Theorem 3.3 [7]. For any real numbers $s, p, q$ such that $0 \leq q \leq s \leq p$ and which do not belong to $\mathbb{N}+1 / 4$, and for any $r$ such that $0 \leq r \leq s$, the space $\mathbf{H}_{\rho}^{s}(\Lambda) \cap \mathbf{H}_{\rho, 0}^{r}(\Lambda)$ is contained in $\mathscr{K}_{\theta}\left(\mathbf{H}_{\rho}^{p}(\Lambda) \cap \mathbf{H}_{\rho, 0}^{r}(\Lambda), \mathbf{H}_{\rho}^{q}(\Lambda) \cap \mathbf{H}_{\rho, 0}^{\min (r, q)}(\Lambda)\right)$, with $\theta=(s-p) /(q-p)$.

3.3. Some properties of the dual spaces. In order to sharpen the previous results on Hilbert interpolation, we need some properties of the duality between the spaces $\mathbf{H}_{\rho, 0}^{s}(\Lambda)$. Let us introduce, for any real number $s>0$, the dual space $\mathbf{H}_{\rho}^{-s}(\Lambda)$ of $\mathbf{H}_{\rho, 0}^{s}(\Lambda)$. In the following, we shall always identify $\mathbf{L}_{\rho}^{2}(\Lambda)$ with 
its dual space. As a consequence, differentiation in the space of distribution $\mathscr{D}^{\prime}(\Lambda)$ is defined as follows:

$$
\forall f \in \mathscr{D}^{\prime}(\Lambda), \forall \varphi \in \mathscr{D}(\Lambda), \quad\langle d f / d x, \varphi\rangle=-\langle f,(1 / \rho)[d(\varphi \rho) / d x]\rangle .
$$

Obviously, this definition coincides with the classical notion of differentiation for regular functions.

The following lemma is then an easy consequence of [8, Chapter 1, Proposition 2.1].

Lemma 3.3. For any real number $s \geq 0,\left[\mathbf{H}_{\rho, 0}^{s}(\Lambda), \mathbf{H}_{\rho}^{-s}(\Lambda)\right]_{1 / 2}$ is equal to $\mathbf{L}_{\rho}^{2}(\Lambda)$.

Now we can prove

Lemma 3.4. For any real numbers $p$ and $q, p \geq q \geq 0$, such that $p$ and $q$ do not belong to $\mathbb{N}+1 / 4$, and for any $\theta$ in $(0,1)$ such that

$$
s=\theta p+(1-\theta) q \notin \mathbb{N}+1 / 4
$$

we have

$$
\left[\mathbf{H}_{\rho}^{-q}(\Lambda), \mathbf{H}_{\rho}^{-p}(\Lambda)\right]_{\theta}=\mathbf{H}_{\rho}^{-s}(\Lambda)
$$

Proof. Recall that, under the conditions of the lemma, Theorem 3.1 states that

$$
\left[\mathbf{H}_{\rho, 0}^{p}(\Lambda), \mathbf{H}_{\rho, 0}^{q}(\Lambda)\right]_{1-\theta}=\mathbf{H}_{\rho, 0}^{s}(\Lambda) .
$$

Using now the theorem of duality (see [8, Chapter 1, Theorem 6.2]), we deduce (3.24) from the definition of the spaces $\mathbf{H}_{\rho}^{-r}(\Lambda)$ for $r>0$.

Using now the technique of [8, Chapter 1, Theorem 12.3], we deduce from the previous results:

Lemma 3.5. For any positive real numbers $p$ and $q$ such that $p$ and $q$ do not belong to $\mathbb{N}+1 / 4$, and for any $\theta$ in $(0,1)$ such that

$$
s=(1-\theta) p-\theta q \notin \mathbb{N}+1 / 4 \text { and }-s \notin \mathbb{N}+1 / 4,
$$

we have

$$
\left[\mathbf{H}_{\rho, 0}^{p}(\Lambda), \mathbf{H}_{\rho}^{-q}(\Lambda)\right]_{\theta}= \begin{cases}\mathbf{H}_{\rho, 0}^{s}(\Lambda) & \text { if } s \geq 0 \\ \mathbf{H}_{\rho}^{s}(\Lambda) & \text { if } s<0 .\end{cases}
$$

We are going to prove now that, in the case where $m$ is an integer, the elements of the space $\mathbf{H}_{\rho}^{-m}(\Lambda)$ are derivatives of some functions in $\mathbf{L}_{\rho}^{2}(\Lambda)$. More precisely, let us introduce the space $\mathscr{H}_{\rho}^{-m}(\Lambda)=\left\{d^{m} f / d x^{m} ; f \in \mathbf{L}_{\rho}^{2}(\Lambda)\right\}$.

Theorem 3.4. For any integer $m \geq 0$, the space $\mathscr{H}_{\rho}^{-m}(\Lambda)$ coincides with the space $\mathbf{H}_{\rho}^{-m}(\Lambda)$.

Proof. Let $u$ be in $\mathbf{H}_{\rho, 0}^{m}(\Lambda)$; it is an easy consequence of Hardy's theorem, and has been proved in [1, Lemma 3.2], that $(1 / \rho)\left[d^{m}(u \rho) / d x^{m}\right]$ belongs to 
$\mathbf{L}_{\rho}^{2}(\Lambda)$. It follows that, for any $f$ in $\mathbf{L}_{\rho}^{2}(\Lambda)$,

$$
\int_{\Lambda} f(x)\left[d^{m}(u \rho) / d x^{m}\right](x) d x \leq C\|f\|_{0, \rho}\|u\|_{m, \rho} .
$$

We conclude from this bound, and from (3.22), that $d^{m} f / d x^{m}$ is in $\mathbf{H}_{\rho}^{-m}(\Lambda)$. This proves that $\mathscr{H}_{\rho}^{-m}(\Lambda) \subset \mathbf{H}_{\rho}^{-m}(\Lambda)$.

Conversely, it follows from [1, Lemma 3.2 and Corollary 3.1] that the mapping $u \rightarrow(1 / \rho)\left[d^{m}(u \rho) / d x^{m}\right]$ defines an isomorphism from $\mathbf{H}_{\rho, 0}^{m}(\Lambda)$ onto a closed subspace of $\mathbf{L}_{\rho}^{2}(\Lambda)$. Hence, for any $\mathbf{L}$ in $\mathbf{H}_{\rho}^{-m}(\Lambda)$, there exists an element $\widetilde{L}$, defined on this closed subspace, such that

$$
\forall u \in \mathbf{H}_{\rho, 0}^{m}(\Lambda), \quad\langle L, u\rangle=\left\langle\widetilde{L},(1 / \rho)\left[d^{m}(u \rho) / d x^{m}\right]\right\rangle .
$$

We deduce from the Hahn-Banach theorem that $\widetilde{L}$ can be extended into an element of the dual space of $\mathbf{L}_{\rho}^{2}(\Lambda)$, whence of $\mathbf{L}_{\rho}^{2}(\Lambda)$. Let $f$ be that element; it is then obvious that $L$ and $d^{m} f / d x^{m}$ coincide on $\mathbf{H}_{\rho, 0}^{m}(\Lambda)$. This proves that $\mathbf{H}_{\rho}^{-m}(\Lambda) \subset \mathscr{H}_{\rho}^{-m}(\Lambda)$.

3.4. The case of Hilbert interpolation. In this section we prove a more precise result concerning Hilbert interpolation between weighted Sobolev spaces.

Theorem 3.5. For any integers $s$ and $p$ such that $0<s<p$, the space $\left[\mathbf{H}_{\rho}^{p}(\Lambda), \mathbf{L}_{\rho}^{2}(\Lambda)\right]_{(p-s) / p}$ is equal to $\mathbf{H}_{\rho}^{s}(\Lambda)$.

Proof. It is an easy consequence of well-known results on interpolation between standard Sobolev spaces that

$$
\left[\mathbf{H}_{\rho}^{p}(\Lambda), \mathbf{L}_{\rho}^{2}(\Lambda)\right]_{(p-s) / p} \subset\left[\mathbf{H}^{p}(\Lambda), \mathbf{L}^{2}(\Lambda)\right]_{(p-s) / p}=\mathbf{H}^{s}(\Lambda),
$$

so that from Theorem 3.2

$$
\left[\mathbf{H}_{\rho}^{p}(\Lambda), \mathbf{L}_{\rho}^{2}(\Lambda)\right]_{(p-s) / p} \subset \mathbf{H}_{\rho}^{s-1}(\Lambda) .
$$

In addition, the operator $d^{p} / d x^{p}$ is linear continuous from the space $\mathbf{H}_{\rho}^{p}(\Lambda)$ into $\mathbf{L}_{\rho}^{2}(\Lambda)$ and, from Theorem 3.4, it is also linear continuous from $\mathbf{L}_{\rho}^{2}(\Lambda)$ into the dual space $\mathbf{H}_{\rho}^{-p}(\Lambda)$. It follows from the principal theorem of interpolation [8, Chapter 1, Theorem 5.1] that $d^{p} / d x^{p}$ is linear continuous from the space $\left[\mathbf{H}_{\rho}^{p}(\Lambda), \mathbf{L}_{\rho}^{2}(\Lambda)\right]_{(p-s) / p}$ into $\left[\mathbf{L}_{\rho}^{2}(\Lambda), \mathbf{H}_{\rho}^{-p}(\Lambda)\right]_{(p-s) / p}$. The space coincides with $\mathbf{H}_{\rho}^{s-p}(\Lambda)$, so that we can state

$$
\left[\mathbf{H}_{\rho}^{p}(\Lambda), \mathbf{L}_{\rho}^{2}(\Lambda)\right]_{(p-s) / p} \subset\left\{f \in \mathbf{H}_{\rho}^{s-1}(\Lambda) ; d^{p} f / d x^{p} \in \mathbf{H}_{\rho}^{s-p}(\Lambda)\right\} .
$$

We deduce from Theorem 3.4 that, if $f$ is an element of $\left[\mathbf{H}_{\rho}^{p}(\Lambda), \mathbf{L}_{\rho}^{2}(\Lambda)\right]_{(p-s) / p}$, there exists an element $g$ of $\mathbf{L}_{\rho}^{2}(\Lambda)$ such that $d^{p} f / d x^{p}=d^{p-s} g / d x^{p-s}$. It is an easy matter to check that $d^{s} f / d x^{s}-g$ is a polynomial in $\mathbb{P}_{p-s-1}$ and that $d^{s} f / d x^{s}$ is in fact an element of $\mathbf{L}_{\rho}^{2}(\Lambda)$. We derive from (3.28)

$$
\left[\mathbf{H}_{\rho}^{p}(\Lambda), \mathbf{L}_{\rho}^{2}(\Lambda)\right]_{(p-s) / p} \subset\left\{f \in \mathbf{H}_{\rho}^{s-1}(\Lambda) ; d^{s} f / d x^{s} \in \mathbf{L}_{\rho}^{2}(\Lambda)\right\}=\mathbf{H}_{\rho}^{s}(\Lambda) .
$$

This imbedding, and (3.6), give the theorem. 
The reiteration theorem, together with the definition of the spaces $\mathbf{H}_{\rho}^{s}(\Lambda)$, gives the following general result:

Theorem 3.6. For any real numbers $s, p, q$ such that $0 \leq q \leq s \leq p, q<p$, we have

$$
\mathbf{H}_{\rho}^{s}(\Lambda)=\left[\mathbf{H}_{\rho}^{\rho}(\Lambda), \mathbf{H}_{\rho}^{q}(\Lambda)\right]_{(p-s) /(p-q)} .
$$

Moreover, we can state

Theorem 3.7. For any real numbers $s, p, q$ not in $\mathbb{N}+1 / 4$ such that $0 \leq q \leq$ $s \leq p$, and for any integer $r$ such that $r \leq s$, we have

$$
\mathbf{H}_{\rho}^{s}(\Lambda) \cap \mathbf{H}_{\rho, 0}^{r}(\Lambda)=\left[\mathbf{H}_{\rho}^{p}(\Lambda) \cap \mathbf{H}_{\rho, 0}^{r}(\Lambda), \mathbf{H}_{\rho}^{q}(\Lambda) \cap \mathbf{H}_{\rho, 0}^{\min (r, q)}(\Lambda)\right]_{(p-s) /(p-q)} .
$$

Proof. It is an easy matter to deduce from (3.29) that

$$
\begin{gathered}
{\left[\mathbf{H}_{\rho}^{p}(\Lambda) \cap \mathbf{H}_{\rho, 0}^{r}(\Lambda), \mathbf{H}_{\rho}^{q}(\Lambda) \cap \mathbf{H}_{\rho, 0}^{\min (r, q)}(\Lambda)\right]_{(p-s) /(p-q)}} \\
\subset\left[\mathbf{H}_{\rho}^{p}(\Lambda), \mathbf{H}_{\rho}^{q}(\Lambda)\right]_{(p-s) /(p-q)}=\mathbf{H}_{\rho}^{s}(\Lambda) .
\end{gathered}
$$

In addition, we also have, if $r \leq q$,

$$
\left[\mathbf{H}_{\rho}^{p}(\Lambda) \cap \mathbf{H}_{\rho, 0}^{r}(\Lambda), \mathbf{H}_{\rho}^{q}(\Lambda) \cap \mathbf{H}_{\rho, 0}^{r}(\Lambda)\right]_{(p-s) /(p-q)} \subset \mathbf{H}_{\rho, 0}^{r}(\Lambda) .
$$

Hence we first deduce the imbedding, in the case where $r \leq q$,

$$
\begin{aligned}
{\left[\mathbf{H}_{\rho}^{p}(\Lambda)\right.} & \left.\cap \mathbf{H}_{\rho, 0}^{r}(\Lambda), \mathbf{H}_{\rho}^{q}(\Lambda) \cap \mathbf{H}_{\rho, 0}^{\min (r, q)}(\Lambda)\right]_{(p-s) /(p-q)} \\
& \subset \mathbf{H}_{\rho}^{s}(\Lambda) \cap \mathbf{H}_{\rho, 0}^{r}(\Lambda) .
\end{aligned}
$$

Furthermore, if $r>q$, it is an easy matter to check that the space $\mathscr{C}^{\infty}(\Lambda) \cap$ $\mathbf{H}_{\rho, 0}^{r}(\Lambda)$ is dense in the spaces $\mathbf{H}_{\rho}^{p}(\Lambda) \cap \mathbf{H}_{\rho, 0}^{r}(\Lambda)$ and $\mathbf{H}_{\rho, 0}^{q}(\Lambda)$; therefore, $\mathscr{C}^{\infty}(\Lambda) \cap \mathbf{H}_{\rho, 0}^{r}(\Lambda)$ is also dense in the space

$$
\left[\mathbf{H}_{\rho}^{p}(\Lambda) \cap \mathbf{H}_{\rho, 0}^{r}(\Lambda), \mathbf{H}_{\rho, 0}^{q}(\Lambda)\right]_{(p-s) /(p-q)},
$$

and (3.31) is also satisfied for any $r$.

Conversely, assume now that neither $s-1 / 4$ nor $p-1 / 4$ nor $q-1 / 4$ are integers. Let $u$ be an element of $\mathbf{H}_{\rho}^{s}(\Lambda) \cap \mathbf{H}_{\rho, 0}^{r}(\Lambda)$. We define the polynomial $\rho$ of $\mathbb{P}_{2(s-1 / 4)}-1$ such that $u-\rho$ is an element of $\mathbf{H}_{\rho, 0}^{s}(\Lambda)$. It is an easy consequence of Theorem 3.1 that $\mu$ exists and satisfies

$$
\forall \sigma>0, \quad\|\rho\|_{\sigma, \rho} \leq C\|u\|_{s, \rho} \text { and } \mu \in \mathbf{H}_{\rho, 0}^{r}(\Lambda) .
$$

We deduce from equality (3.5) that the element $u-\rho$ belongs to the space $\left[\mathbf{H}_{\rho, 0}^{p}(\Lambda), \mathbf{H}_{\rho, 0}^{q}(\Lambda)\right]_{(p-s) /(p-q)}$, hence

$$
u-\mu \in\left[\mathbf{H}_{\rho}^{p}(\Lambda) \cap \mathbf{H}_{\rho, 0}^{r}(\Lambda), \mathbf{H}_{\rho}^{q}(\Lambda) \cap \mathbf{H}_{\rho, 0}^{\min (r, q)}(\Lambda)\right]_{(p-s) /(p-q)} .
$$

From (3.32) we derive that

$$
u \in\left[\mathbf{H}_{\rho}^{p}(\Lambda) \cap \mathbf{H}_{\rho, 0}^{r}(\Lambda), \mathbf{H}_{\rho}^{q}(\Lambda) \cap \mathbf{H}_{\rho, 0}^{\min (r, q)}(\Lambda)\right]_{(p-s) /(p-q)},
$$


which proves the converse inclusion of $(3.31)$ whenever $s-1 / 4, p-1 / 4$, or $q-1 / 4$ are not integers.

\section{APPROXIMATION RESULTS FOR PROJECTION OPERATORS IN THE WEIGHTED SOBOLEV SPACES}

Let $p$ be a nonnegative real number not in $\mathbb{N}+1 / 4$. By virtue of (3.5), we can define a new scalar product over $\mathbf{H}_{\rho, 0}^{p}(\Lambda)$. Indeed, for any $p$ such that $2 p$ is not in $\mathbb{N}+1 / 4, \mathbf{H}_{\rho, 0}^{p}(\Lambda)$ can be obtained by interpolation between $\mathbf{L}_{\rho}^{2}(\Lambda)$ and $\mathbf{H}_{\rho, 0}^{2 p}(\Lambda)$ of index $1 / 2$, and for any $p$ such that $2 p$ is in $\mathbb{N}+1 / 4, \mathbf{H}_{\rho, 0}^{p}(\Lambda)$ can be obtained by interpolation between $\mathbf{L}_{\rho}^{2}(\Lambda)$ and $\mathbf{H}_{\rho, 0}^{3 p}(\Lambda)$ of index $1 / 3$.

If we consider domain operator interpolation, we can deduce the existence of a self-adjoint operator $\Theta_{p, \rho}$ such that

-the domain $\mathbf{D}\left(\boldsymbol{\Theta}_{p, \rho}\right)$ of the operator $\Theta_{p, \rho}$ in $\mathbf{L}_{\rho}^{2}(\Lambda)$ is $\mathbf{H}_{\rho, 0}^{p}(\Lambda)$;

-if $2 p$ does not belong to $\mathbb{N}+1 / 4$, the domain $\mathbf{D}\left(\Theta_{p, \rho}^{2}\right)$ of the operator $\Theta_{p, \rho}^{2}$ in $\mathbf{L}_{\rho}^{2}(\Lambda)$ is $\mathbf{H}_{\rho, 0}^{2 p}(\Lambda)$; if $2 p$ belongs to $\mathbb{N}+1 / 4$, the domain $\mathbf{D}\left(\Theta_{p, \rho}^{3}\right)$ of the operator $\Theta_{p, \rho}^{3}$ in $\mathbf{L}_{\rho}^{2}(\Lambda)$ is $\mathbf{H}_{\rho, 0}^{3 p}(\Lambda)$.

Moreover, the bilinear form

$$
(u, v) \rightarrow(((u, v)))_{p, \rho}=(u, v)_{\rho}+\left(\Theta_{p, \rho} u, \Theta_{p, \rho} v\right)_{\rho}
$$

is a scalar product whose associated norm, denoted by $\||\cdot|\| \|_{p, \rho}$, is equivalent to the one defined in (3.4).

We are going to examine the properties of the projection operators related to the scalar product defined in (4.1). The interest in considering this kind of projection operator will appear when we shall analyze the approximation properties they satisfy in lower-order norms.

We first choose $p$ in $\mathbb{N}$, and we define $\mathbf{P}_{p, N}$ as the orthogonal projection operator from $\mathbf{H}_{\rho, 0}^{p}(\Lambda)$ onto $\mathbb{P}_{N}^{p}$ with respect to the scalar product defined in (4.1).

Lemma 4.1. For any $\varphi \in \mathbf{H}_{\rho}^{\sigma}(\Lambda) \cap \mathbf{H}_{\rho, 0}^{p}(\Lambda)$, with $\sigma \geq p$, we have

$$
\forall \nu, \quad 0 \leq \nu \leq p, \quad\left\|\varphi-\mathbf{P}_{p, N} \varphi\right\|_{\nu, \rho} \leq C N^{\nu-\sigma}\|\varphi\|_{\sigma, \rho} .
$$

Remark 4.1. The estimate in the case $\nu=p=0$ has been proved in [4], and the case $0 \leq \nu \leq p=1$ is analyzed in [12] (note that the dependence of the constant $C$ in (4.2) with respect to $\sigma$ is such that there exist two constants $C_{1}$ and $C_{2}, 0<C_{1}<C_{2}$ such that $C_{1} \sigma ! \leq C(\sigma) \leq C_{2} \sigma$ !). Moreover, it is proved that no optimal bound is possible for $\mathbf{H}_{\rho, 0}^{\nu}(\Lambda)$-norms with $\nu>p$. Indeed, for example, the best possible estimate is

$$
\left\|\varphi-\mathbf{P}_{0, N} \varphi\right\|_{\nu, \rho} \leq C N^{2 \nu-\sigma}\|\varphi\|_{\sigma, \rho} .
$$

It is often necessary (see $[5,2,11,18]$ and $(5.13))$ to obtain optimal results in higher norms. 
Proof of Lemma 4.1. We prove (4.2) for $\nu=p$ by induction over $p$ in $\mathbb{N}$. In Remark 4.1 we have recalled that the result is well known for $p=0$. Assume, then, that for any $\varphi$ in $\mathbf{H}_{\rho}^{\sigma}(\Lambda) \cap \mathbf{H}_{\rho, 0}^{p-1}(\Lambda)$ with $\sigma \leq p-1$ we have

$$
\forall \nu, \quad 0 \leq \nu \leq p-1, \quad\left\|\varphi-\mathbf{P}_{p-1, N} \varphi\right\|_{\nu, \rho} \leq C N^{\nu-\sigma}\|\varphi\|_{\sigma, \rho} .
$$

For the same reason as in $\S 2$, we assume that

$$
N>2(p-1)
$$

(note again that the asymptotic result $(4.2)$ is trivial for $N \leq 2(p-1)$ if we take the constant $C$ large enough).

Let $\varphi$ be an element of $\mathbf{H}_{\rho, 0}^{p}(\Lambda)$; then $\varphi^{\prime}$ belongs to $\mathbf{H}_{\rho, 0}^{p-1}(\Lambda)$, and since $\varphi(-1)=\varphi(-1)=0$, we have

$$
\alpha=\int_{-1}^{1} \mathbf{P}_{p-1, N-1}\left(\varphi^{\prime}\right)(t) d t=\int_{-1}^{1}\left[\mathbf{P}_{p-1, N-1}\left(\varphi^{\prime}\right)-\varphi^{\prime}\right](t) d t .
$$

Here, one cannot prove that $\alpha$ is equal to 0 , but from the Cauchy-Schwarz inequality we find

$$
\begin{aligned}
|\alpha| & \leq\left(\int_{-1}^{1}\left[\mathbf{P}_{p-1, N-1}\left(\varphi^{\prime}\right)-\varphi^{\prime}\right]^{2}(t) \rho(t) d t\right)^{1 / 2}\left(\int_{-1}^{1} \rho^{-1}(t) d t\right)^{1 / 2} \\
& \leq C\left\|\mathbf{P}_{p-1, N-1}\left(\varphi^{\prime}\right)-\varphi^{\prime}\right\|_{0, \rho},
\end{aligned}
$$

so that, from the induction hypothesis (4.3) we obtain

$$
|\alpha| \leq C N^{1-\sigma}\left\|\varphi^{\prime}\right\|_{\sigma-1, \rho} .
$$

Finally, we deduce that the primitive function

$$
R_{N}(x)=\int_{-1}^{x}\left[\mathbf{P}_{p-1, N-1}\left(\varphi^{\prime}\right)(t)-\left(\alpha\left(1-t^{2}\right)^{p-1}\right) /\left(\int_{-1}^{1}\left(1-s^{2}\right)^{p-1} d s\right)\right] d t
$$

is a polynomial in $\mathbb{P}_{N}^{p}$ (note that we have used here the hypothesis (4.4)). From the Poincaré-Friedrichs inequality (see [1, Corollary 2.1]) we derive

$$
\left\|\varphi-R_{N}\right\|_{p, \rho} \leq\left\|\left(\varphi-R_{N}\right)^{\prime}\right\|_{p-1, \rho},
$$

and the induction hypothesis (4.3), together with (4.5), gives

$$
\left\|\varphi-R_{N}\right\|_{p, \rho} \leq C\left(N^{(p-1)-(\sigma-1)}+N^{(1-\sigma)}\right)\left\|\varphi^{\prime}\right\|_{\sigma-1, \rho} \leq C N^{p-\sigma}\|\varphi\|_{\sigma, \rho} .
$$

From the equivalence of the norms $\|\cdot\|_{p, \rho}$ and $\|\cdot \cdot\|_{p, \rho}$ we derive that

$$
\left\|\varphi-R_{N} \mid\right\|_{p, \rho} \leq C N^{p-\sigma}\|\varphi\|_{\sigma, \rho} .
$$

Since the definition of $\mathbf{P}_{p, N}$ yields the relation

$$
\left\|\left|\varphi-\mathbf{P}_{p, N}(\varphi)\right|\right\|_{p, \rho}=\inf _{\varphi_{N} \in \mathbb{P}_{N}^{p}}|| \varphi-\varphi_{N}\left|\left\|_{p, \rho} \leq\right\|\left\|\varphi-R_{N} \mid\right\|_{p, \rho},\right.
$$

we obtain for any $\varphi$ in $\mathbf{H}_{\rho}^{\sigma}(\Lambda) \cap \mathbf{H}_{\rho, 0}^{p}(\Lambda)$

$$
\left\|\varphi-\mathbf{P}_{p, N}(\varphi)\right\|\left\|_{p, \rho} \leq C N^{p-\sigma}\right\| \varphi \|_{\sigma, \rho} .
$$

This proves the induction hypothesis for $\nu=p$. 
Next, to derive the case $\nu=0$, we use the abstract duality result of the appendix. With $\mathbf{Y}_{0}=\mathbf{L}_{\rho}^{2}(\Lambda), \mathbf{Y}=\mathbf{D}\left(\Theta_{p, \rho}^{2}\right), \mathbf{Z}=\mathbf{H}_{\rho}^{2 p}(\Lambda) \cap \mathbf{H}_{\rho, 0}^{p}(\Lambda)$, and $\mathbf{X}=\mathbf{H}_{\rho, 0}^{p}(\Lambda)$, we obtain by using (4.6) that

$$
\begin{aligned}
\left\|\varphi-\mathbf{P}_{p, N}(\varphi)\right\|_{0, \rho} & \leq C N^{p-\sigma}\|\varphi\|_{\sigma, \rho} \sup _{\psi \in \mathbf{H}_{\rho}^{2 p}(\Lambda) \cap \mathbf{H}_{\rho, 0}^{p}(\Lambda)} \frac{\left\|\psi-\mathbf{P}_{p, N}(\psi)\right\|_{p, \rho}}{\|\psi\|_{2 p, \rho}} \\
& \leq C N^{-\sigma}\|\varphi\|_{\sigma, \rho} .
\end{aligned}
$$

Now, from the two estimates

$$
\begin{aligned}
& \forall \sigma \geq p, \quad\left\|\varphi-\mathbf{P}_{p, N}(\varphi)\right\|_{p, \rho} \leq C N^{p-\sigma}\|\varphi\|_{\sigma, \rho}, \\
& \forall \sigma \geq p, \quad\left\|\varphi-\mathbf{P}_{p, N}(\varphi)\right\|_{0, \rho} \leq C N^{-\sigma}\|\varphi\|_{\sigma, \rho},
\end{aligned}
$$

valid for any $\varphi \in \mathbf{H}_{\rho}^{\sigma}(\Lambda) \cap \mathbf{H}_{0, \rho}^{p}(\Lambda)$, we obtain for any $\theta \in(0,1)$

$$
\left\|\varphi-\mathbf{P}_{p, N}(\varphi)\right\|_{\left[\mathbf{H}_{\rho, 0}^{p}(\Lambda), \mathbf{L}_{\rho}^{2}(\Lambda)\right]_{\theta}} \leq C N^{\theta p-\sigma}\|\varphi\|_{\sigma, \rho} .
$$

From this result with $\theta=\nu / p$, we deduce, using (3.5), that for any $0 \leq \nu \leq p$ we have

$$
\left\|\varphi-\mathbf{P}_{p, N}(\varphi)\right\|_{\nu, \rho} \leq C N^{\nu-\sigma}\|\varphi\|_{\sigma, \rho},
$$

which completes the induction argument.

As in $\S 2$, we are now interested in the approximation of the spaces $\mathbf{H}_{\rho}^{p}(\Lambda) \cap$ $\mathbf{H}_{\rho, 0}^{s}(\Lambda)$ by elements of $\mathbb{P}_{N}^{s}$, when $s$ and $p$ are integers such that $0 \leq s<p$. From Theorem 3.1 we deduce that there exists a linear continuous operator $\mathbf{A}_{p}$ from $\mathbf{H}_{\rho}^{p}(\Lambda)$ into the subspace of polynomials $\mathbb{P}_{2 p-1}$ such that for any $\varphi$ in $\mathbf{H}_{\rho}^{p}(\Lambda)$ we have

$$
\begin{aligned}
& \varphi-\mathbf{A}_{p} \varphi \in \mathbf{H}_{\rho, 0}^{p}(\Lambda), \quad\left\|\varphi-\mathbf{A}_{p} \varphi\right\|_{p, \rho} \leq C\|\varphi\|_{p, \rho}, \\
& \forall \mu \in \mathbb{R}^{+}, \quad\left\|\mathbf{A}_{p} \varphi\right\|_{\mu, \rho} \leq C(\mu)\|\varphi\|_{p, \rho} .
\end{aligned}
$$

By Lemma 4.1, we have for any $\sigma \geq p$ and any $\varphi$ in $\mathbf{H}_{\rho}^{p}(\Lambda) \cap \mathbf{H}_{\rho, 0}^{s}(\Lambda)$

$$
\left\|\left(\varphi-\mathbf{A}_{p} \varphi\right)-\left(\mathbf{P}_{p, N}\left(\varphi-\mathbf{A}_{p} \varphi\right)\right)\right\|_{\nu, \rho} \leq C N^{\nu-\sigma}\left\|\varphi-\mathbf{A}_{p} \varphi\right\|_{\sigma, \rho} .
$$

We deduce from (4.8) that

$$
\left\|\varphi-\left(\mathbf{A}_{p} \varphi+\mathbf{P}_{p, N}\left(\varphi-\mathbf{A}_{p} \varphi\right)\right)\right\|_{\nu, \rho} \leq C N^{\nu-\sigma}\|\varphi\|_{\sigma, \rho} .
$$

The operator $\widetilde{\mathbf{P}}_{p, N}=\mathbf{A}_{p}+\mathbf{P}_{p, N} \circ\left(\mathbf{I d}-\mathbf{A}_{p}\right)$ from $\mathbf{H}_{\rho}^{p}(\Lambda) \cap \mathbf{H}_{\rho, 0}^{s}(\Lambda)$ onto $\mathbb{P}_{N} \cap$ $\mathbf{H}_{\rho, 0}^{s}(\Lambda)$ is such that

$$
\left\|\varphi-\widetilde{\mathbf{P}}_{p, N} \varphi\right\|_{\nu, \rho} \leq C N^{\nu-\sigma}\|\varphi\|_{\sigma, \rho} .
$$

As noted in [18], the operator $\widetilde{\mathbf{P}}_{p, N}$ preserves the traces of any element of 
$\mathbf{H}_{\rho}^{p}(\Lambda)$. This proves that in fact $\widetilde{\mathbf{P}}_{p, N}$ is independent of $s$. We have proved Lemma 4.2. For any $\varphi \in \mathbf{H}_{\rho}^{\sigma}(\Lambda) \cap \mathbf{H}_{\rho, 0}^{s}(\Lambda)$ with $\sigma \geq p$, we have

$$
\forall \nu, \quad 0 \leq \nu \leq p, \quad\left\|\varphi-\widetilde{\mathbf{P}}_{p, N} \varphi\right\|_{\nu, \rho} \leq C N^{\nu-\sigma}\|\varphi\|_{\sigma, \rho} .
$$

Remark 4.2. The operator $\widetilde{\mathbf{P}}_{p, N}$ is not defined as a projection operator for a scalar product. This may be a disadvantage in some applications (see [2]), but we note that we can define a new scalar product over $\Lambda$ that induces a norm equivalent to the original one by using Theorems 3.6 and 3.7. More precisely, for example in the case where $s=0$, we recall that the space $\mathbf{H}_{\rho}^{2 p}(\Lambda)$ is the domain of a positive, self-adjoint operator $\widetilde{\Omega}$ in $\mathbf{L}_{\rho}^{2}(\Lambda)$ and that the domain of $\Omega=(\widetilde{\Omega})^{1 / 2}$ is then $\mathbf{H}_{\rho}^{p}(\Lambda)$. The space $\mathbf{H}_{\rho}^{p}(\Lambda)$ can be provided with the scalar product

$$
((u, v))=(u, v)_{\rho}+(\Omega u, \Omega v)_{\rho} .
$$

If we define the projection operator $\mathbf{P}_{p, N}$ on $\mathbb{P}_{N}$ with respect to this scalar product, we obtain from (4.10) that

$$
\left\|u-\mathbf{P}_{p, N} u\right\|_{p, \rho} \leq C N^{p-\sigma}\|u\|_{\sigma, \rho} .
$$

Using now the abstract duality result with $\mathbf{Y}_{0}=\mathbf{L}_{\rho}^{2}(\Lambda), \mathbf{Z}=\mathbf{H}_{\rho}^{2 p}(\Lambda)=\mathbf{Y}$, and $\mathbf{X}=\mathbf{H}_{\rho}^{p}(\Lambda)$, we deduce that

$$
\left\|u-\mathbf{P}_{p, N} u\right\|_{0, \rho} \leq C N^{-\sigma}\|u\|_{\sigma, \rho} .
$$

The complete set $0 \leq \nu \leq p$ is recovered by an interpolation argument that relies upon Theorem 3.6. We also obtain for any $u$ in $\mathbf{H}_{\rho}^{\sigma}(\Lambda)$ with $\sigma \geq p$ that

$$
\forall \nu, \quad 0 \leq \nu \leq p, \quad\left\|u-\mathbf{P}_{p, N} u\right\|_{\nu, \rho} \leq C N^{\nu-\sigma}\|u\|_{\sigma, \rho} .
$$

We now prove an analogue of (4.2) for nonintegral values of $p$ such that $p-1 / 4$ is not in $\mathbb{N}$. Here, the two cases $p-\bar{p}<1 / 4$ and $p-\bar{p}>1 / 4$ must be distinguished.

We begin with the case $p-\bar{p}>1 / 4$ and take $\varphi$ in $\mathbf{H}_{\rho}^{\sigma}(\Lambda) \cap \mathbf{H}_{\rho, 0}^{p}(\Lambda)$ with $\sigma \geq \bar{p}+1$. It is a consequence of Theorem 3.1 that $\varphi$ is, in fact, in $\mathbf{H}_{\rho, 0}^{\bar{p}+1}(\Lambda)$. Using now Lemma 4.1 , we obtain

$$
\inf _{\varphi_{N} \in \mathbb{P}_{N}^{\overline{+}+1}}\left\|\varphi-\varphi_{N}\right\|_{p, \rho} \leq C N^{p-\sigma}\|\varphi\|_{\sigma, \rho}
$$

for any $\sigma \geq \bar{p}+1$ (see (4.2) for $\mathbf{P}_{\bar{p}+1, N}$ in the case $\nu=p$ ). Since we have $\mathbb{P}_{N}^{\bar{p}+1}=\mathbb{P}_{N}^{p}$, we deduce that

$$
\forall \sigma \geq \bar{p}+1, \quad \inf _{\varphi_{N} \in \mathbb{P}_{N}^{p}}\left\|\varphi-\varphi_{N}\right\|_{p, \rho} \leq C N^{p-\sigma}\|\varphi\|_{\sigma, \rho} .
$$

Consider next the case $p-\bar{p}<1 / 4$ and take $\varphi \in \mathbf{H}_{\rho}^{\sigma}(\Lambda) \cap \mathbf{H}_{\rho, 0}^{p}(\Lambda), \sigma \geq \bar{p}+1$. Using now Lemma 4.2 with $\widetilde{\mathbf{P}}_{\bar{p}+1, N}$ gives

$$
\inf _{\varphi_{N} \in \mathbb{P}_{N}^{\bar{p}}}\left\|\varphi-\varphi_{N}\right\|_{p, \rho} \leq C N^{p-\sigma}\|\varphi\|_{\sigma, \rho}
$$


(see (4.10) for $\widetilde{\mathbf{P}}_{p+1, N}$ with $\nu=p$ ), so that, for any $\sigma \geq \bar{p}+1$,

$$
\forall \sigma \geq \bar{p}+1, \quad \inf _{\varphi_{N} \in \mathbb{P}_{N}^{p}}\left\|\varphi-\varphi_{N}\right\|_{p, \rho} \leq C N^{p-\sigma}\|\varphi\|_{\sigma, \rho} .
$$

From (4.11) and (4.12), the projection operator $\mathbf{P}_{p, N}$ from $\mathbf{H}_{0}^{p}(\Lambda)$ onto $\mathbb{P}_{N}^{p}$ with respect to the scalar product defined in (4.1) satisfies the following estimate, for any $\varphi \in \mathbf{H}_{\rho}^{\sigma}(\Lambda) \cap \mathbf{H}_{\rho, 0}^{p}(\Lambda), \sigma \geq \bar{p}+1$,

$$
\left\|\varphi-\mathbf{P}_{p, N} \varphi\right\|_{p, \rho} \leq C N^{p-\sigma}\|\varphi\|_{\sigma, \rho}
$$

Trivially, we also have

$$
\left\|\varphi-\mathbf{P}_{p, N} \varphi\right\|_{p, \rho} \leq C\|\varphi\|_{p, \rho} .
$$

A simple interpolation argument, that relies upon Theorem 3.7, now gives, for any $\sigma \geq p$ and any $\varphi \in \mathbf{H}_{\rho}^{\sigma}(\Lambda) \cap \mathbf{H}_{\rho, 0}^{p}(\Lambda)$,

$$
\left\|\varphi-\mathbf{P}_{p, N} \varphi\right\|_{p, \rho} \leq C N^{p-\sigma}\|\varphi\|_{\sigma, \rho}
$$

The same techniques as in $\S 2$, which rely on the abstract duality result and an interpolation argument, yield for any $\varphi \in \mathbf{H}_{\rho}^{\sigma}(\Lambda) \cap \mathbf{H}_{\rho, 0}^{p}(\Lambda), \sigma \geq p$, and any $\nu, 0 \leq \nu \leq p$, that

$$
\left\|\varphi-\mathbf{P}_{p, N}(\varphi)\right\|_{\nu, \rho} \leq C N^{\nu-\sigma}\|\varphi\|_{\sigma, \rho} .
$$

Following the same lines as in the proof of Lemma 4.2, we can prove

Theorem 4.1. Let $p$ and $s$ be two real numbers such that $p \notin \mathbb{N}+1 / 4,0 \leq$ $s \leq p$. There exists an operator $\Pi_{p, N}^{s, 0}$ from $\mathbf{H}_{\rho}^{p}(\Lambda) \cap \mathbf{H}_{\rho, 0}^{s}(\Lambda)$ onto $\mathbb{P}_{N}^{s}$ such that, for any $\varphi \in \mathbf{H}_{\rho}^{\sigma}(\Lambda) \cap \mathbf{H}_{\rho, 0}^{s}(\Lambda)$ with $\sigma \geq p$, we have

$$
\forall \nu, \quad 0 \leq \nu \leq p, \quad\left\|\varphi-\Pi_{p, N}^{s, 0} \varphi\right\|_{\nu, \rho} \leq C N^{\nu-\sigma}\|\varphi\|_{\sigma, \rho} .
$$

Remark 4.3. Similar results concerning the existence of an operator from the closed subspace of those elements of $\mathbf{H}_{\rho}^{p}(\Lambda)$ for which only some of the derivatives of order $<p-1 / 4$ vanish on the boundary of $\Lambda$ onto the subspace of polynomials of $\mathbb{P}_{N}$ satisfying the same boundary conditions can be obtained following the same lines.

Remark 4.4. We point out that the previous operators can all be seen as orthogonal projection operators with respect to some scalar product (recall Remark 4.2). In the general case, it seems difficult to exhibit exactly the analytic form of this scalar product, but the previous remark will be essential in a forthcoming paper.

\section{AN APPLicAtion}

5.1. Definition of the problem. In order to explain how the previous results can be applied, we consider the approximate solution of the very simple problem: find $\psi$ defined on $\Lambda$ such that

$$
d^{4} \psi / d x^{4}=f \quad \text { on } \Lambda, \quad \psi( \pm 1)=(d \psi / d x)( \pm 1)=0
$$


(This problem is a first step towards the numerical approximation of the Stokes problem in the $\psi$-formulation; see $[10,11]$.)

As in $\S 3, \rho$ will denote the Chebyshev weight; cf. $\S 3.1$. We want to prove the following

Theorem 5.1. For any $f \in \mathbf{H}_{\rho}^{-2}(\Lambda)$ there exists one and only one solution $\psi$ of the problem (5.1) in the space $\mathbf{H}_{\rho, 0}^{2}(\Lambda)$.

This theorem is a consequence of the Lax-Milgram lemma and the following lemma.

Lemma 5.1. There exist three positive constants $\alpha, \beta, \gamma$ such that for any $(\varphi, \psi)$ $\in \mathbf{H}_{\rho}^{2}(\Lambda) \times \mathbf{H}_{0, \rho}^{2}(\Lambda)$

$$
\begin{gathered}
\left(\psi^{\prime \prime},(\psi \rho)^{\prime \prime}\right) \geq \alpha\|\psi\|_{2, \rho}^{2}, \\
\left(\varphi^{\prime \prime},(\psi \rho)^{\prime \prime}\right) \leq \gamma\left\|\varphi^{\prime \prime}\right\|_{0, \rho}\left\|\psi^{\prime \prime}\right\|_{0, \rho} .
\end{gathered}
$$

Proof. (i) We first obtain the following inequalities, valid for any $\psi$ in $\mathscr{D}(\Lambda)$,

$$
\begin{aligned}
\int_{\Lambda} \psi^{\prime \prime}( & x)(\psi \rho)^{\prime \prime}(x) d x \\
= & \int_{\Lambda}\left(\psi^{\prime \prime}\right)^{2}(x) \rho(x) d x+2 \int_{\Lambda} \psi^{\prime \prime}(x) \psi^{\prime}(x) \rho^{\prime}(x) d x \\
& +\int_{\Lambda} \psi^{\prime \prime}(x) \psi(x) \rho^{\prime \prime}(x) d x \\
= & \int_{\Lambda}\left(\psi^{\prime \prime}\right)^{2}(x) \rho(x) d x+\int_{\Lambda}\left(\psi^{\prime 2}\right)^{\prime}(x) \rho^{\prime}(x) d x+\int_{\Lambda}\left(\psi^{\prime}\right)^{\prime}(x) \psi(x) \rho^{\prime \prime}(x) d x \\
= & \int_{\Lambda}\left(\psi^{\prime \prime}\right)^{2}(x) \rho(x) d x-\int_{\Lambda} \psi^{\prime 2}(x) \rho^{\prime \prime}(x) d x-\int_{\Lambda} \psi^{\prime}(x)\left(\psi \rho^{\prime \prime}\right)^{\prime}(x) d x \\
= & \int_{\Lambda}\left(\psi^{\prime \prime}\right)^{2}(x) \rho(x) d x-2 \int_{\Lambda} \psi^{\prime 2}(x) \rho^{\prime \prime}(x) d x \\
& +(1 / 2) \int_{\Lambda} \psi^{2}(x) \rho^{\prime \prime \prime \prime}(x) d x .
\end{aligned}
$$

Note that

$$
\begin{aligned}
\rho^{\prime \prime}(x) & =\left(1+2 x^{2}\right) \rho^{5}(x), \\
\rho^{\prime \prime \prime \prime}(x) & =\left(9+72 x^{2}+24 x^{4}\right) \rho^{9}(x),
\end{aligned}
$$

from which we derive

$$
\begin{aligned}
\int_{\Lambda} \psi^{\prime \prime}(x)(\psi \rho)^{\prime \prime}(x) d x \\
=\int_{\Lambda}\left(\psi^{\prime \prime}\right)^{2}(x) \rho(x) d x-2 \int_{\Lambda} \psi^{\prime 2}(x)\left(1+2 x^{2}\right) \rho^{5}(x) d x \\
\quad+(1 / 2) \int_{\Lambda} \psi^{2}(x)\left(9+72 x^{2}+24 x^{4}\right) \rho^{9}(x) d x
\end{aligned}
$$


Now set

$$
\begin{aligned}
P=\int_{\Lambda}\left(\psi^{\prime \prime}\right. & (x) \rho(x)+2 x \psi^{\prime}(x) \rho^{3}(x) \\
& \left.+\left(2 x^{2}+10^{-2}\right) \psi(x) \rho^{5}(x)\right)^{2} \rho^{-1}(x) d x .
\end{aligned}
$$

We note that $P \geq 0$; moreover, an easy calculation gives

$$
\begin{aligned}
P= & \int_{\Lambda} \psi^{\prime \prime}(x)(\psi \rho)^{\prime \prime}(x) d x-2 \cdot 10^{-2} \int_{\Lambda} \psi^{\prime 2}(x) \rho^{5}(x) d x \\
& -\int_{\Lambda}\left(5 \cdot 78 x^{2}+0.4699\right) \psi^{2}(x) \rho^{9}(x) d x,
\end{aligned}
$$

so that

$$
\int_{\Lambda} \psi^{\prime 2}(x) \rho^{5}(x) d x \leq 50 \int_{\Lambda} \psi^{\prime \prime}(x)(\psi \rho)^{\prime \prime}(x) d x .
$$

From this we derive that

$$
\int_{\Lambda} \psi^{\prime 2}(x)\left(1+2 x^{2}\right) \rho^{5}(x) d x \leq 150 \int_{\Lambda} \psi^{\prime \prime}(x)(\psi \rho)^{\prime \prime}(x) d x,
$$

and using this inequality in (5.4), we obtain a constant $c>0$ such that

$$
\int_{\Lambda} \psi^{\prime \prime}(x)(\psi \rho)^{\prime \prime}(x) d x \geq c \int_{\Lambda}\left(\psi^{\prime \prime}\right)^{2}(x) \rho(x) d x .
$$

Inequality (5.2) then follows as a consequence of the Poincaré-Friedrichs inequality.

(ii) Finally, let us note that for any $(\varphi, \psi)$ in $\mathbf{H}_{\rho}^{2}(\Lambda) \times \mathbf{H}_{0, \rho}^{2}(\Lambda)$ we have

$$
\begin{aligned}
\left(\varphi^{\prime \prime},(\psi \rho)^{\prime \prime}\right)= & \left(\varphi^{\prime \prime}, \psi^{\prime \prime}\right)_{\rho}+2 \int_{\Lambda} \varphi^{\prime \prime}(x) \psi^{\prime}(x) \rho^{\prime}(x) d x \\
& +\int_{\Lambda} \varphi^{\prime \prime}(x) \psi(x) \rho^{\prime \prime}(x) d x
\end{aligned}
$$

We easily derive

$$
\left|\left(\varphi^{\prime \prime}, \psi^{\prime \prime}\right)_{\rho}\right| \leq\left\|\varphi^{\prime \prime}\right\|_{0, \rho}\left\|\psi^{\prime \prime}\right\|_{0, \rho} .
$$

Let us examine the second term in (5.5). We have

$$
\begin{aligned}
& \left|\int_{\Lambda} \varphi^{\prime \prime}(x) \psi^{\prime}(x) \rho^{\prime}(x) d x\right| \\
& \quad=\left|\int_{\Lambda} \varphi^{\prime \prime}(x)\left(\psi^{\prime} \rho^{\prime} \rho^{-1}\right)(x) \rho(x) d x\right| \\
& \leq\left|\int_{\Lambda}\left(\varphi^{\prime \prime}\right)^{2}(x) \rho(x) d x\right|^{1 / 2}\left|\int_{\Lambda}\left(\psi^{\prime} \rho^{\prime}\right)^{2}(x) \rho^{-1}(x) d x\right|^{1 / 2} .
\end{aligned}
$$

Since $\rho^{\prime 2}(x) \rho^{-1}(x)=x^{2} \rho^{5}(x)$, as a consequence of Hardy's inequality we derive that (see [1, Lemma 2.2])

$$
\left|\int_{\Lambda} \varphi^{\prime \prime}(x) \psi^{\prime}(x) \rho^{\prime}(x) d x\right| \leq C\left\|\varphi^{\prime \prime}\right\|_{0, \rho}\left\|\psi^{\prime \prime}\right\|_{0, \rho} .
$$


In a similar way we obtain

$$
\left|\int_{\Lambda} \varphi^{\prime \prime}(x) \psi(x) \rho^{\prime \prime}(x) d x\right| \leq C\left\|\varphi^{\prime \prime}\right\|_{0, \rho}\left\|\psi^{\prime \prime}\right\|_{0, \rho},
$$

so that $(5.3)$ is a consequence of $(5.5)-(5.8)$.

Proof of Theorem 5.1. Let $f$ be in $\mathbf{H}_{\rho}^{-2}(\Lambda)$. Problem (5.1) is equivalent to finding $\psi$ in $\mathbf{H}_{\rho, 0}^{2}(\Lambda)$ such that, for any $\varphi$ in $\mathbf{H}_{\rho, 0}^{2}(\Lambda)$,

$$
\int_{\Lambda} \psi^{\prime \prime}(x)(\varphi \rho)^{\prime \prime}(x) d x=\langle f, \varphi\rangle
$$

The bilinear form $a$, defined for any $(\chi, \varphi)$ in $\mathbf{H}_{\rho, 0}^{2}(\Lambda)^{2}$ by

$$
a(\chi, \varphi)=\int_{\Lambda} \chi^{\prime \prime}(x)(\varphi \rho)^{\prime \prime}(x) d x
$$

is continuous and elliptic on $\mathbf{H}_{\rho, 0}^{2}(\Lambda)$ (see Lemma 5.1). The Lax-Milgram lemma gives the existence and uniqueness of a solution of (5.9), hence of (5.1).

Remark 5.1. We point out that problem (5.1) is defined for any $f$ in the space $\mathbf{H}_{\rho}^{-2}(\Lambda)$, hence, a priori, the right-hand side of (5.9) cannot be written as $\int_{\Lambda} f(x) \varphi(x) \rho d x$. This formulation is valid if we assume that $f$ is more regular, at least in $\mathbf{L}_{\rho}^{2}(\Lambda)$. In the general case, we know from Theorem 3.4 that there exists a function $g$ of $\mathbf{L}_{\rho}^{2}(\Lambda)$ such that $f=g^{\prime \prime}$, and

$$
\forall \varphi \in \mathbf{H}_{\rho, 0}^{1}(\Lambda), \quad\langle f, \varphi\rangle=\int_{\Lambda} g(x)(\varphi \rho)^{\prime \prime} d x .
$$

5.2. Approximation of problem (5.1). We are interested in the approximation of the solution of problem (5.1) by a polynomial of degree $\leq N$. We use a Galerkin spectral method (we refer to [13] and to [15] for numerical implementations and more details on the method). From (5.9) we derive a discrete problem: find $\psi_{N}$ in $\mathbb{P}_{N}^{2}$ such that, for any $\varphi$ in $\mathbb{P}_{N}^{2}$,

$$
\int_{\Lambda} \psi_{N}^{\prime \prime}(\varphi \rho)^{\prime \prime} d x=\langle f, \varphi\rangle .
$$

From Lemma 5.1 we know that problem (5.11) is well posed in the sense that there exists one and only one solution to it. Moreover, we derive from (5.9) and (5.11) that

$$
a\left(\psi-\psi_{N}, \varphi\right)=0 \text { for any } \varphi \text { in } \mathbb{P}_{N}^{2},
$$

so that (recall that $\Pi_{2, N}^{2,0} \psi \in \mathbb{P}_{N}^{2}$ )

$$
a\left(\psi-\psi_{N}, \psi-\psi_{N}\right)=a\left(\psi-\psi_{N}, \psi-\Pi_{2, N}^{2,0} \psi\right) .
$$

By Theorem 4.1 and Lemma 5.1, we obtain the following result. 
Theorem 5.2. For any $f$ in $\mathbf{H}_{\rho}^{-2}(\Lambda)$, there exists one and only one solution $\psi_{N}$ to problem (5.11); moreover, if the solution $\psi$ of (5.1) belongs to $\mathbf{H}_{\rho}^{\sigma}(\Lambda) \cap$ $\mathbf{H}_{\rho, 0}^{2}(\Lambda)$, we have

$$
\left\|\psi-\psi_{N}\right\|_{2, \rho} \leq C N^{2-\sigma}\|\psi\|_{\sigma, \rho} .
$$

Remark 5.2. The previous estimate is optimal, since no polynomial of $\mathbb{P}_{N}^{2}$ is asymptotically nearer to the solution $\psi$ than the solution $\psi_{N}$ of the approximate problem.

Remark 5.3. The previous theorem will be extended in a future work, in order to consider collocation and tau pseudospectral approximations of one-dimensional fourth-order problems. Such methods are much more efficient from a computational point of view.

\section{APPENDIX. AN ABSTRACT DUALITY RESULT}

Consider two Hilbert spaces $\mathbf{X}$ and $\mathbf{Y}_{0}, \mathbf{X} \subset \mathbf{Y}_{0}$, with a continuous imbedding. Moreover, suppose that $\mathbf{X}$ is dense in $\mathbf{Y}_{0}$. It is classical (see, e.g., [17]) to prove that there exists an unbounded operator $\Theta$, self-adjoint and positive definite, such that $\mathbf{X}$ is the domain $\mathbf{D}\left(\Theta ; \mathbf{Y}_{0}\right)$ of $\Theta$ in $\mathbf{Y}_{0}$. Denote by $(\cdot, \cdot)_{0}$ the scalar product in $\mathbf{Y}_{0}$ and by $\|\cdot\|_{0}$ its norm. We can define a new scalar product on $\mathbf{X}$ equivalent to the initial one by

$$
((u, v))=(u, v)_{0}+(\Theta u, \Theta v)_{0},
$$

such that the associated norm

$$
\|u\|=\left[\|u\|_{0}^{2}+\|\Theta u\|_{0}^{2}\right]^{1 / 2}
$$

is equivalent to the initial one. Define the space

$$
\mathbf{Y}=\mathbf{D}\left(\Theta^{2} ; \mathbf{Y}_{0}\right)
$$

and equip it with the norm

$$
\|u\|_{\mathbf{Y}}=\left[\|u\|_{0}^{2}+\left\|\Theta^{2} u\right\|_{0}^{2}\right]^{1 / 2}
$$

We now turn to the approximation problem. Consider a family $\left(\mathbf{X}_{N}\right)_{N}$ of closed subspaces of $\mathbf{X}$ indexed by $N \in \mathbb{N}$. We denote by $\Pi_{N}$ the projection operator from $\mathbf{X}$ onto $\mathbf{X}_{N}$ for the scalar product defined in (A.1).

Theorem A.1. Let $\mathbf{Z}$ be a Hilbert space such that $\mathbf{Y} \subset \mathbf{Z} \subset \mathbf{X}$, and such that $\mathbf{Z}$ is dense in $\mathbf{X}$. The following approximation result holds for any $u$ in $\mathbf{X}$ :

$$
\left\|u-\Pi_{N} u\right\|_{0} \leq \sup _{\varphi \in \mathbf{Z}} \frac{\left\|\varphi-\Pi_{N} \varphi\right\|}{\|\varphi\|_{\mathbf{Z}}}\left\|u-\Pi_{N} u\right\| .
$$

Proof. Denote by $\tilde{u}$ the difference $u-\Pi_{N} u$. We have

$$
\left\|u-\Pi_{N} u\right\|_{0}^{2}=\left(u-\Pi_{N} u, \tilde{u}\right)_{0}=\left(u-\Pi_{N} u, \Theta^{2}\left(\Theta^{-2}\right) \tilde{u}\right)_{0} .
$$


Since $\Theta$ is self-adjoint and positive, we derive

$$
\begin{aligned}
\| u- & \Pi_{n} u \|_{0}^{2}=\left(\Theta\left(u-\Pi_{N} u\right), \Theta\left(\Theta^{-2}\right) \tilde{u}\right)_{0} \\
& \leq\left(\Theta\left(u-\Pi_{N} u\right), \Theta\left(\Theta^{-2}\right) \tilde{u}\right)_{0}+\left(\Theta^{-1}\left(u-\Pi_{N} u\right), \Theta^{-1}\left(u-\Pi_{N} u\right)\right)_{0} \\
& \leq\left(\Theta\left(u-\Pi_{n} u\right), \Theta\left(\Theta^{-2}\right) \tilde{u}\right)_{0}+\left(\left(u-\Pi_{N} u\right),\left(\Theta^{-2}\right) \widetilde{u}\right)_{0} .
\end{aligned}
$$

Therefore, from the definition of the scalar product (A.1) we have

$$
\left\|u-\Pi_{N} u\right\|_{0}^{2} \leq\left(\left(u-\Pi_{N} u,\left(\Theta^{-2}\right) \tilde{u}\right)\right) .
$$

The definition of the projection operator $\Pi_{N}$ yields

$$
\forall \varphi_{N} \in \mathbf{X}_{N}, \quad\left\|u-\Pi_{N} u\right\|_{0}^{2} \leq\left(\left(u-\Pi_{N} u,\left(\Theta^{-2}\right) \tilde{u}-\varphi_{N}\right)\right) .
$$

The choice of $\varphi_{N}=\Pi_{N}\left(\left(\Theta^{-2}\right) \tilde{u}\right)$ gives

$$
\left\|u-\Pi_{N} u\right\|_{0}^{2} \leq\left(\left(u-\Pi_{N} u,\left(\Theta^{-2}\right) \tilde{u}-\Pi_{N}\left[\left(\Theta^{-2}\right) \tilde{u}\right]\right)\right) ;
$$

we now derive

$$
\left\|u-\Pi_{N} u\right\|_{0}^{2} \leq c\left\|u-\Pi_{N} u\right\|\left\|\left(\Theta^{-2}\right) \tilde{u}-\Pi_{N}\left[\left(\Theta^{-2}\right) \tilde{u}\right]\right\|,
$$

whence we obtain

$$
\left\|u-\Pi_{N} u\right\|_{0}^{2} \leq c\left\|u-\Pi_{N} u\right\| \sup _{\varphi \in \mathbf{Z}} \frac{\left\|\varphi-\Pi_{N} \varphi\right\|}{\|\varphi\|_{\mathbf{Z}}}\left\|\left(\Theta^{-2}\right) \tilde{u}\right\|_{\mathbf{Z}}
$$

It is an easy matter to note that

$$
\left\|\left(\Theta^{-2}\right) \tilde{u}\right\|_{\mathbf{Z}} \leq\left\|\left(\Theta^{-2}\right) \tilde{u}\right\|_{\mathbf{Y}}
$$

Also, since $\Theta$ is positive, $\Theta^{-1}$ is bounded, and we obtain

$$
\left\|\left(\Theta^{-2}\right) \tilde{u}\right\|_{\mathbf{Z}} \leq c\|\tilde{u}\|_{0}=c\left\|u-\Pi_{N} u\right\|_{0},
$$

from which the theorem follows.

\section{ACKNOWLEDGMENTS}

The author would like to thank C. Bernardi, P. Grisvard, and F. Murat for valuable and enjoyable discussions. This research has been supported in part by the National Aeronautics and Space Administration under NASA Contract No. NAS1-17070 while the author was in residence at the Institute for Computer Applications in Sciences and Engineering, NASA Langley Research Center, Hampton, Virginia.

\section{BIBLIOGRAPHY}

1. C. Bernardi and Y. Maday, Properties of some weighted Sobolev spaces and application to spectral approximation, SIAM J. Numer. Anal. 26 (1989), 769-829.

2. __ Some spectral approximation of one-dimensional fourth-order problems, J. Approx. Theory (to appear). 
3. C. Bernardi, Y. Maday, and B. Métivet, Spectral approximation of the periodic nonperiodic Navier-Stokes equations, Numer. Math. 51 (1987), 655-700.

4. C. Canuto and A. Quarteroni, Approximation results for orthogonal polynomials in Sobolev spaces, Math. Comp. 38 (1982), 67-86.

5. __ Variational methods in the theoretical analysis of spectral approximation, Proc. Sympos. on Spectral Methods for P.D.E. (R. G. Voigt, D. Gottlieb, and M. Y. Hussaini, eds.), SIAM, Philadelphia, 1984, pp. 55-78.

6. P. Grisvard, Espaces intermédiaires entre espaces de Sobolev avec poids, Ann. Scuola Norm. Sup. Pisa 17 (1963).

7. _ Private communication.

8. J. L. Lions and E. Magenes, Problèmes aux limites non homogènes et applications, Vol. I, Dunod, 1968.

9. J. L. Lions and J. Peetre, Sur une classe d'espaces d'interpolation, Inst. Hautes Études Sci. Publ. Math. 19 (1964), 5-68.

10. Y. Maday and B. Métivet, Estimation d'erreur pour l'approximation des équations de Stokes par une méthode spectrale, Rech. Aérospat. 5 (1983), 237-244.

11. _ Chebyshev spectral approximation of Navier-Stokes equations in a two-dimensional domain, $\mathrm{M}^{2} \mathrm{AN} 21$ (1986), 93-123.

12. Y. Maday and A. Quarteroni, Legendre and Chebyshev spectral approximation of Burgers' equation, Numer. Math. 37 (1981), 321-332.

13. F. Montigny-Rannou, Etude d'un problème de stabilité par résolution de l'équation de Orr-Sommerfeld. Application à la détermination d'un champs de vitesse initial turbulent, ONERA-RT 4/3419 AY, 1980.

14. J. Necas, Les méthodes directes en théorie des équations elliptiques, Édition de l'Académie Tchécoslovaque des Sciences, Prague, 1967.

15. S. A. Orszag, Accurate solution of the Orr-Sommerfeld stability equation, J. Fluid Mech. $\mathbf{5 0}$ (1971), 689-703.

16. A. Quarteroni, Blended Fourier and Chebyshev interpolation, J. Approx. Theory 51 (1987), 115-126.

17. F. Riesz and B. Sz.-Nagy, Leçons d'analyse fonctionelle, Akad. Kiado, Budapest, 1952.

18. G. Sacchi Landriani and H. Vandeven, Polynomial approximation of divergence-free functions, Math. Comp. 52 (1989), 103-130.

19. E. Tadmor, The exponential accuracy of Fourier and Chebyshev differencing methods, SIAM J. Numer. Anal. 23 (1986), 1-10.

Laboratoire d'Analyse Numérique, Universite Pierre et Marie Curie, Tour 55-65, $5^{\circ}$ étage, 4 place Jussieu, F-75252 Paris Cedex 05, France 